\title{
Induced Pluripotent Stem Cell-Differentiated Chondrocytes Repair Cartilage Defect in a Rabbit Osteoarthritis Model
}

\author{
Yu-Hsun Chang, ${ }^{1}$ Kun-Chi Wu, ${ }^{2}$ and Dah-Ching Ding $\mathbb{D}^{3,4,5}$ \\ ${ }^{1}$ Department of Pediatrics, Hualien Tzu Chi Hospital, Buddhist Tzu Chi Medical Foundation, and Tzu Chi University, \\ Hualien, Taiwan \\ ${ }^{2}$ Department of Orthopedics, Hualien Tzu Chi Hospital, Buddhist Tzu Chi Medical Foundation, and Tzu Chi University, \\ Hualien, Taiwan \\ ${ }^{3}$ Department of Obstetrics and Gynecology, Hualien Tzu Chi Hospital, Buddhist Tzu Chi Medical Foundation, and Tzu \\ Chi University, Hualien, Taiwan \\ ${ }^{4}$ Department of Research, Hualien Tzu Chi Hospital, Buddhist Tzu Chi Medical Foundation, and Tzu Chi University, \\ Hualien, Taiwan \\ ${ }^{5}$ Institute of Medical Sciences, Tzu Chi University, Hualien, Taiwan
}

Correspondence should be addressed to Dah-Ching Ding; dah1003@yahoo.com.tw

Received 2 July 2020; Revised 8 October 2020; Accepted 19 October 2020; Published 10 November 2020

Academic Editor: Valdo Jose Dias Da Silva

Copyright (c) $2020 \mathrm{Yu}$-Hsun Chang et al. This is an open access article distributed under the Creative Commons Attribution License, which permits unrestricted use, distribution, and reproduction in any medium, provided the original work is properly cited.

\begin{abstract}
The aim of this study was to explore the therapeutic effect of iPSC-mesenchymal stem cell (MSC)-derived chondrocytes in a rabbit osteoarthritis (OA) model. The iPSCs were characterized by gene expressions, immunostaining of pluripotent markers, and in vivo teratoma formation. iPSC-differentiated MSCs were characterized by flow cytometry and trilineage differentiation. A rabbit OA model was established by the transection of the anterior cruciate ligament. The therapeutic effect of transplanted iPSC-MSCchondrocytes on the OA was evaluated by the histology, immunostaining, and qPCR of defective cartilage. The results showed iPSC could express pluripotency markers such as OCT4, SOX2, and NANOG and form an embryoid body and a teratoma. After differentiation of iPSCs for 30 days, MSCs were established. The iPSC-MSC could express typical MSC markers such as CD29, CD44, CD90, CD105, and HLA-ABC. They could differentiate into adipocytes, osteocytes, and chondrocytes. In this model, iPSC-MSC-chondrocytes significantly improved the histology and ICRS (International Cartilage Repair Society) scores. The transplanted cartilage expressed less IL- $1 \beta$, TNF- $\alpha$, and MMP13 than control cartilage. In conclusion, the iPSCs we derived might represent an emerging source for differentiated MSC-chondrocyte and might rescue cartilage defects through its antiinflammatory and anti-catabolic effects.
\end{abstract}

\section{Introduction}

Osteoarthritis (OA) is characterized by a gradual articular cartilage loss due to unknown causes [1]. Chondrocytes would produce proteolytic enzymes, including metalloproteinases and aggrecanases, to destroy collagen and proteoglycans [2]; gradually loss of cartilage may lead to joint space narrowing and underlying bone exposure [3]. OA has an impact on the health of both men and women worldwide and about $10 \%-15 \%$ of adults over 60 years old have some degree of OA. It is becoming an increasingly important disease, ranked as a cause for second disability [4].

The current symptoms' control treatments of OA include both pharmacological and nonpharmacological approaches [5] as well as knee joint replacement in cases of severe cartilage destruction [6]. However, these treatment modalities still lack a definite efficacy. Regeneration therapies, including autologous serum injection, platelet-rich plasma, and mesenchymal stem cell (MSC) transplantation, have been proposed [7-10]. Mechanisms of such therapies are anti-inflammation 
and chondrocyte regeneration by MSCs [11, 12]. However, cell source, number of derived cells, and invasive harvesting procedures represent a limitation to their use [13].

Characteristics of induced pluripotent stem cells (iPSCs) are the same as embryonic stem cells. After introducing Oct4, sox2, Nanog, c-myc, and klf4 genes, the cells can turn back to the embryonic stage and have pluripotent characteristics [14, 15]. Their use for therapeutic purposes has also emerged, such as for retinal regeneration [16]. In addition, iPSCs have been found to differentiate into chondrocytes via mesenchymal progenitors with embryoid body formation [17-19]. However, the use of progenitor cells, which may be contaminated with pluripotent stem cells, has caused tumor generation [20]. Therefore, the elimination of pluripotency cell contamination is critical for successful iPSC therapy [21].

As chondrocytes represent a cell type with terminal differentiation, the iPSC-differentiated chondrocytes might be associated with a decreased chance of tumor formation after transplantation. The other drawbacks of iPSC included instability of the induced phenotype and possible accelerated senescence [22]. Several ways to conquer accelerated senescence are in combination with the SV40 large T antigen (SV40 LT) and/or hTERT [23] or knockdown p53 expression [24].

The aim of the present study was to use iPSCdifferentiated MSC-derived chondrocytes to repair cartilage defects with OA and explore the therapeutic mechanism.

\section{Materials and Methods}

2.1. The Culture of iPSCs. The iPSC line was developed from normal human epidermal keratinocytes (NHEK) cell line bought from PromoCell (Heidelberg, Germany). The whole study protocol was followed by the institutional guidelines and approved by the Research Ethics Committee, Hualien Tzu Chi Hospital (IRB 104-46-A).

iPSC cells were generated using Sendai virus reprogramming [25]. Briefly, the NHEK cell line was maintained in culture plates precoated with EpiLife medium (Invitrogen, Carlsbad, CA, USA) and supplemented with EpiLife human keratinocytes growth supplement (HKGS) (Invitrogen) at $37^{\circ} \mathrm{C}$ in a $5 \% \mathrm{CO} 2$ incubator. We changed the medium every 48 hours and subcultured them when the cells reached $75 \%$ of confluence. We used a nonintegrating system to introduce four genes (CytoTune ${ }^{\mathrm{TM}}$-iPS 2.0 Sendai reprogramming kit [Invitrogen, Carlsbad, CA, USA, Cat. No. A16517]). The system used Sendai virus particles to deliver polycistronic KLF4-OCT3/4-SOX2, CMYC, and KLF4. NHEK cells were seeded at a density of $5 \times 10^{4}$ cells per well $(34.8 \mathrm{~mm}$ in diameter) in EpiLife medium. We changed the medium every other day until the cells reached a confluence of $40 \%$ with small clusters formation at 2-3 days. Then, we transduced the Sendai virus vector at an MOI of 4:4:2 (KLF4OCT3/4-SOX2:CMYC: KLF4) in the medium. After 24 hours, the cells were washed with $1 \mathrm{X}$ Dulbecco's PBS (-Ca2+/Mg2+) (Gibco, Massachusetts, United States) and fed with fresh EpiLife medium containing HKGS. At 7 days posttransfection, we passaged cells onto vitronectin (rhVTN-N) (Gibco, Massachusetts, United States)-coated 6-well plates at a density of $2 \times 10^{4}$ cells/well. We fed every day with chemically defined Essential 8 medium (Gibco, Massachusetts, United States) and $10 \mu \mathrm{M}$ Y-27632 (Merck Millipore Massachusetts, United States). After 12 days of transduction, we manually isolated positive TRA-1-60 or TRA-1-81 colonies under live cell staining. We passaged the iPSC with the EDTA method (0.5 M EDTA, pH 8.0, Invitrogen, Carlsbad, CA, USA) [26].

The resulted iPSCs were cultured on MEF feeder cells [27] with a culture media which consisted of $80 \%(v / v)$ knockout (KO) DMEM, 20\% ( $v / v)$ KO serum replacement, $2 \mathrm{mM}$ L-glutamine, $10 \mathrm{mM}$ nonessential amino acid (Invitrogen, Waltham, MA, USA), and $4 \mathrm{ng} / \mathrm{mL}$ basic fibroblast growth factor (bFGF).

2.2. Pluripotency of iPSC. iPSCs' pluripotency was evaluated by immunostaining and RT-PCR for Oct4, Sox2, and Nanog (pluripotency markers). In vitro differentiation was demonstrated with markers of three germ layers [brachyury (mesoderm), Tuj-1 (ectoderm), and AFP (endoderm)] by means of embryoid body formation, for 5 days. In order to evaluate the iPSCs' pluripotency phenotype, we performed in vivo differentiation. Specifically, iPSCs were subcutaneously injected in nonobese diabetes and severe combined immunodeficient (NOD-SCID) mice. After 3 months, the xenograft tumors were examined. The three germ layers' differentiation was demonstrated by RT-PCR. The latter included endoderm (GATA4), mesoderm (Hand1 and GATA6), and ectoderm (Tubb3, MAP2, and GFAP).

2.3. Protocol for Differentiation into iPSC-Derived MSC Cells. We modified a previously published protocol to differentiate iPSC into MSC [28]. We dissociated undifferentiated iPSC with $4 \mathrm{mg} / \mathrm{ml}$ of collagenase I to remove MEFs and any differentiated cells. The resulting iPSC colonies were dissociated by manual pipetting and then placed onto Matrigelcoated dishes. Subsequently, iPSC media was changed to the MSC culture medium. The MSC culture medium contained DMEM-low glucose (DMEM-LG, Gibco, Grand Island, NY, USA) with 10\% fetal bovine serum (FBS, Biological Industry, Kibbutz, Israel), 100 units/mL of Penicillin and Streptomycin (Gibco). The MSC medium was changed every day for a total of 5 days. Cells were then detached from the Matrigel-coated dishes using 2.5\% trypsin/0.23 mM ethylenediaminetetraacetic acid (EDTA, Sigma) at $37^{\circ} \mathrm{C}$ for $10 \mathrm{~min}$. Cells were subsequently cultured on uncoated culture dishes. The medium was changed every 3 days. When reaching a $90 \%$ confluence of 7 days of culture, cells were passaged with a $1: 3$ ratio. The morphology of iPSCs and iPSC-MSCs was observed by a microscope image (Nikon, Tokyo, Japan). This step took nearly one month.

2.4. Flow Cytometry of iPSC-MSC Cells. MSC-specific surface markers were evaluated and characterized by flow cytometry. iPSC-derived MSCs were detached using 2 mM EDTA in PBS and washed. They were then incubated with the candidate antibody conjugated with phycoerythrin (PE) or fluorescein isothiocyanate (FITC). Specifically, candidate antibodies included the following: CD14, CD19, CD24, CD29, CD34, 
CD44, CD45, CD56, CD73, CD90, CD105, CD117, CD133, HLA-ABC, and HLA-DR (BD, Pharmingen). Becton Dickinson flow cytometer (Vantage SE, Becton Dickinson, San Jose, CA, USA) was used for the analysis.

2.5. In vitro Adipogenic and Osteogenic Differentiation of iPSCMSCs. To evaluate the iPSC-derived MSCs' in vitroadipogenic and osteogenic potential, cells were detached from the dish and underwent progeny differentiation. Adipogenic differentiation was induced by culturing $3 \times 10^{5} \mathrm{cells} / \mathrm{cm}^{2}$ in an adipogenic medium. The latter consisted of DMEM supplemented with $10 \% \mathrm{FBS}, 1 \mathrm{mmol} / \mathrm{L}$ dexamethasone, $5 \mathrm{mg} / \mathrm{mL}$ insulin, $0.5 \mathrm{mmol} / \mathrm{L}$ isobutylmethylxanthine, and $60 \mathrm{mmol} / \mathrm{L}$ indomethacin (Sigma). The medium was changed every 3 days for 3 weeks. Osteogenic differentiation was induced by culturing $6 \times 10^{4}$ cells $/ \mathrm{cm}^{2}$ in the osteogenic differentiation medium. Such a medium consisted of DMEM supplemented with $10 \%$ FBS, $0.1 \mathrm{mmol} / \mathrm{L}$ dexamethasone, $10 \mathrm{mmol} / \mathrm{L} \beta$-glycerol phosphate, and $50 \mathrm{mmol} / \mathrm{L}$ ascorbate (Sigma). Adipogenic differentiation was evaluated based on the presence of lipid droplets in the cells through $0.3 \%$ Oil Red O stain (Sigma) for $15 \mathrm{~min}$ at room temperature. Osteogenic differentiation was evaluated by mineralization of calcium accumulation by Alizarin Red $\mathrm{S}$ staining (Sigma).

2.6. In vitro Chondrogenic Differentiation of iPSC-MSC. To study the chondrogenic potential of iPSC-derived MSCs, cells were detached from the dish, and chondrogenic differentiation took place in a micromass method [25]. Briefly, $1 \times 10^{5}$ iPSCderived MSCs were grown in the chondrogenic medium. The latter consisted of DMEM, 10\% FBS, $10 \mathrm{ng} / \mathrm{mL}$ transforming growth factor- $\beta 1$ (TGF- $\beta 1$ ), $50 \mu \mathrm{g} / \mathrm{mL}$ of ascorbic acid-2phosphate, and $6.25 \mu \mathrm{g} / \mathrm{mL}$ of insulin. The medium was changed every 3 days. The chondrogenic medium was changed every other day for 21 days. Micromasses were formed after differentiation for 21 days and sent for histology, immunostaining, and gene expression analysis.

2.7. In vivo Tumor Xenografts. iPSCs were detached by mechanical cutting, and the resulting sheets were resuspended in Matrigel with PBS (BD, 1:1). The iPSCs obtained were kept on ice for less than $60 \mathrm{~min}$ in order to maintain viability. The cells were then injected subcutaneously into the back tissue of NOD-SCID mice $(n=3)$. Tumor formation follow-up was performed by palpation up to 3 months. Xenograft tumors were evaluated by gene expressions of three germ layer differentiation, GATA4 (endoderm); HAND1 and GATA6 (mesoderm); and TUBB3, MAP2, and GFAP (ectoderm) and compared to their expressions of iPSCs.

2.8. RT-PCR. In order to clarify the gene expression of pluripotency and differentiation in iPSC, RT-PCR was performed. We also detected the gene expressions of potassium channels in iPSC-MSC and BMSC. In order to extract total ribonucleic acid (RNA) from iPSC-MSCs, BMSC, the micromass $(n=3)$ obtained from the cell cultures or tumor tissue from xenografts $(n=3)$, we used an RNeasy Protect Mini Kit with an efficient on-column RNase-free DNase treatment (Qiagen, Hilden, Germany). RNA was eluted in $30 \mathrm{~mL}$ of RNase-free water. RT-PCR was performed using a Super-
Script III One-Step RT-PCR kit (Invitrogen, Grand Island, NY, USA) according to the manufacturer's instructions.

2.9. Quantitative Real-Time PCR ( $q P C R)$. To confirm the progeny differentiation of the iPSC-derived MSCs, qPCR was utilized. Gene expression of the chondrogenic differentiated cells was assessed for hyaline cartilage markers [i.e., type 2 collagen (COL2A1) and Aggrecan (ACAN)].

The glyceraldehyde 3-phosphate dehydrogenase (GAPDH) was used as an internal control. For quantification, we used the expression of the following genes: FABP4 and PPAR $\gamma$ for adipogenesis, and osteopontin $(O P N)$ and alkaline phosphatase $(A L P L)$ for osteogenesis. For inflammatory markers of transplanted cartilage, rabbit $I L-1 \beta, T N F-\alpha$, and $M M P 13$ were tested. Rabbit type 2 collagen (COL2A) and type 1 collagen $(C O L 1)$ were also tested for hyaline cartilage formation. Gene expression changes from iPSC to MSC were tested for OCT4 (a pluripotency gene) and CD73 (a marker of MSC). Table 1 lists the primer sequences used. As to the qPCR procedures, in brief, real-time PCRs were performed and monitored using FastStart Universal SYBR Green Master (ROX, Roche, Indianapolis, IN, USA) and a qPCR detection system (ABI Step One Plus system, Applied Biosystems, Foster City, CA, USA). Each target gene's expression levels were calculated as $2^{-\Delta \Delta \mathrm{Ct}}$ [29]. Three readings for each experimental sample were obtained for each gene of interest. Additionally, the experiments were repeated in triplicates.

2.10. Immunohistochemistry. In order to investigate cells' production of aggrecan and type 2 collagen, immunostaining with type 2 collagen and aggrecan was performed. Chondrogenesis derived micromasses were fixed in $10 \%$ formalin (Sigma), then washed with graded alcohol (70\%, 95\%, and $100 \%)$ to dehydrate and to make tissue transparent with xylene (EMD, Millipore, USA). Micromasses were subsequently embedded in paraffin, sectioned with $4 \mu \mathrm{m}$ thickness, and mounted on glass slides. Hematoxylin and Eosin (H\&E) staining was performed to evaluate cell morphology. The Safranin $\mathrm{O}$ staining was used to evaluate glycosaminoglycan levels. Finally, immunostaining for type 2 collagen and aggrecan was performed for the detection of related protein expression. Specifically, for immunohistochemistry, tissue sections were predigested with pepsin $(1 \mathrm{mg} / \mathrm{mL}$ in Tris$\mathrm{HCl}, \mathrm{pH} 2.0)$ and incubated with type 2 collagen and aggrecan primary antibody (1:200, EMD; Millipore) for $60 \mathrm{~min}$. Reactivity was detected by a diaminobenzidine tetrahydrochloride substrate. We stained cartilage tissue with an antihuman mitochondria antibody $(1: 200$, Sigma-Aldrich) to detect whether human cells were integrating and regenerating after 12 weeks of transplantation. A mouse anti-rabbit CD45 antibody ( $1: 200$, Bio-Rad, Hercules, CA, USA) was used for detecting rabbit CD45 cells located in cartilage. A digital camera under a light microscope (Nikon, Tokyo, Japan) was used to capture images of the stained sections.

2.11. Animal Experiments. In the present study, we used three 14-month-old New Zealand White rabbits. First, the rabbits' knees received an anterior cruciate ligament transection (ACLT) as previously described procedure [30]. 
TABLE 1: Gene primer sequences used for RT-PCR and qPCR experiments.

\begin{tabular}{|c|c|c|c|}
\hline Gene name & Forward sequence & Reverse sequence & Base pair no \\
\hline Oct4 & CAG TGC CCG AAA CCC ACA C & CAG TGC CCG AAA CCC ACA C & 161 \\
\hline Nanog & AGT CCC AAA GGC AAA CAA CCC ACT TC & TGC TGG AGG CTG AGG TAT TTC TGT CTC & 161 \\
\hline Sox2 & GGG AAA TGG GAG GGG TGC AAA AGA GG & TTG CGT GAG TGT GGA TGG GAT TGG TG & 151 \\
\hline MAP2 & GCA TGA GCT CTT GGC AGG & CCA ATT GAA CCC ATG TAA AGC C & 194 \\
\hline GFAP & AGG GCT GAC ACG TCC AC & GCC TTA GAG GGG AGA GGA G & 132 \\
\hline GATA4 & TCС СТС ТTC ССТ ССТ САA АТ & TCA GCG TGT AAA GGC ATC TG & 194 \\
\hline Tubb3 & CAG AGC AAG AAC AGC AGC TAC TT & GTG AAC TCC ATC TCG TCC ATG CCC TC & 227 \\
\hline Hand1 & TGC CTG AGA AAG AGA ACC AG & ATG GCA GGA TGA ACA AAC AC & 274 \\
\hline GATA6 & CCT CAC TCC ACT CGT GTC TGC & GTC CTG GCT TCT GGA AGT GG & 225 \\
\hline$P P A R r$ & AGC CTC ATG AAG AGC CTT CCA & TCC GGA AGA AAC CCT TGC A & 120 \\
\hline OPN & AGG AGG AGG CAG AGC ACA & CTG GTA TGG CAC AGG TGA TG & 150 \\
\hline$A P A L$ & CCACGTCTTCACATTTGGTG & GCAGTGAAGGGCTTCTTGTC & 96 \\
\hline FABP4 & ATGGGATGGAAAATCAACCA & GTGGAAGTGACGCCTTTCAT & 87 \\
\hline Aggrecan & CGAAACATCACTGAGGGTGA & GCAAACGTGAAGGGCTCCT & 107 \\
\hline Col2A1 & GAGAGGTCTTCCTGGCAAAG & AAGTCCCTGGAAGCCAGAT & 118 \\
\hline$C D 73$ & AGTCCACTGGAGAGTTCCTGCA & TGAGAGGGTCATAACTGGGCAC & 133 \\
\hline OCT4 & CTT GCT GCA GAA GTG GGT GGA GGA & CTG CAG TGT GGG TTT CGG GCA & 169 \\
\hline GAPDH & GGTCTCCTCTGACTTGAACA & GTGAGGGTCTCTCTCTTCCT & 221 \\
\hline KCa1.1 & АСААСАТСТСССССААСС & TCATСАССТТСТТТССААТTС & 310 \\
\hline KCa3.1 & CGGGAACAAGTGAACTCCAT & ACTGGGGAAAGTAGCCTGGT & 239 \\
\hline Kv1.2 & ATGAGAGAATTGGGCCTCCT & СССАСТАТСТТТСССССААТ & 200 \\
\hline Kv1.4 & ACGAGGGCTTTGTGAGAGAA & CACGATGAAGAAGGGGTCAT & 308 \\
\hline Kv1.5 & GTAACGTCAAGGCCAAGAGC & GGGAGGAAAGGAGTGAAAGG & 225 \\
\hline Kv1.6 & CTGGCTTGACCACAGTCTGA & CTGGAGTTTGCCTGAGGAAG & 190 \\
\hline Kv2.1 & GTTGGCCATTCTGCCATACT & GCAAAGTGAAGCCCAGAGAC & 173 \\
\hline Kv4.2 & GCTTGTCATCAATCCСCTTG & TCCAGTATCTGGGCTTTTCC & 102 \\
\hline Kv4.3 & ACGGAGACATGGTGCCTAAG & CCCTGCGTTTATCAGCTCTC & 153 \\
\hline KCNH1 & TGGATTTTGCAAGCTGTCTG & GAGTCTTTGGTGCCTCTTGC & 476 \\
\hline Kir2.1 & AACAGGGAGGTGTGGACAAG & TAACCTGCTCTAGGGCTCCA & 261 \\
\hline Kir2.2 & GAGGCTATCACAGGCTCAGG & CCCCAAGTTAAAAACCAGCA & 183 \\
\hline Kir2.3 & GCTTTGAGCCTGTGGTCTTC & TTGGCTCTGTCCTGAGTGTG & 480 \\
\hline Clcn3 & CATAGGTCAAGCAGAGGGTC & TATTTCCGCAGCAACAGG & 293 \\
\hline SCN9A & GCTCCGAGTCTTCAAGTTGG & GGTTGTTTGCATCAGGGTCT & 446 \\
\hline CACNA1C & AACATCAACAACGCCAACAA & AGGGCAGGACTGTCTTCTGA & 574 \\
\hline GAPDH & CCATCTTCCAGGAGCGAG & GCAGGAGGCATTGCTGAT & 233 \\
\hline rCollagen $2 a$ & CCTGTGCGACGACATAATCTGT & GGTCCTTTAGGTCCTACGATATCCT & 176 \\
\hline rCOL1 & CAGAACGGCCTCAGGTACCA & CAGATCACGTCATCGCACAAC & 101 \\
\hline$r T N F a$ & CTGCACTTCAGGGTGATCG & CTACGTGGGCTAGAGGCTTG & 133 \\
\hline rIL1B & TTGAAGAAGAACCCGTCCTCTG & CTCATACGTGCCAGACAACACC & 128 \\
\hline$r M M P 13$ & AGGAAGACCTCCAGTTTGCAGAG & GCTGCATTCTCCTTCAGGATTC & 85 \\
\hline$r G A P D H$ & TGACGACATCAAGAAGGTGGTG & GAAGGTGGAGGAGTGGGTGTC & 120 \\
\hline
\end{tabular}

Then, they were divided into right knees, $\mathrm{OA}+1.8 \times 10^{8}$ iPSC-MSC-chondrocytes, and left knees, OA with $0.5 \mathrm{~mL}$ of saline $(n=3)$.

2.12. Surgical Procedure of OA Rabbit Model. The Animal Studies Ethics Committee of Tzu Chi University approved the experiment's protocol (105-26-2). We confirmed that all methods were performed in accordance with the relevant guidelines and regulations. Adult (48-60 months old, 3.3$4.6 \mathrm{~kg})$ male New Zealand white rabbits $(n=3)$ were anesthetized by an intramuscular injection of xylazine $(8 \mathrm{mg} / \mathrm{kg})$ and ketamine $(100 \mathrm{mg} / \mathrm{kg})$. Both knees were shaved and disinfected with a beta-iodine solution. A medial parapatellar incision through the skin was made, and an arthrotomy was performed. The patella was dislocated laterally, and the knee was placed in a full flexion manner. A no. 15 blade was used 
to transect the anterior cruciate ligament (ACL). The joint was then irrigated with sterile saline and closed with a running suture of 3-0 vicryl (Ethicon, Johnson \& Johnson, Bridgewater, NJ, USA). Subsequently, a mattress suture of 3-0 nylon (Ethicon) was used to close the skin. All rabbits were returned to their cages after the surgery and allowed to freely move. For pain relief, rabbits received $0.2 \mathrm{mg} / \mathrm{kg}$ of oral meloxicam daily for 7 days. The rabbits after the procedures were well in feeding and living on the following days. Two adult (48-60 months old, 3.3-4.6 kg) male New Zealand white rabbits without any procedure were used as normal control.

\subsection{Treatment of iPSC-MSC-Chondrocytes in OA Rabbit} Model. After 8 weeks from ACLT, the right knee joints of the three rabbits were injected with $0.5 \mathrm{~mL} 0.9 \%$ normal saline (Otsuka, Taipei, Taiwan) mixed with $1.8 \times 10^{8}$ iPSCMSC-chondrocytes $\left(1 \times 10^{6}\right.$ iPSC-MSCs were cultured in a $15 \mathrm{~cm}$ dish with the supplement of the chondrocyte differentiation medium stated for above 3 weeks). As an internal control, at the same time, the left knee joints were injected with $0.5 \mathrm{~mL}$ saline. Subsequently, 12 weeks after cell therapy, we used pentobarbital (3\%) $15-40 \mathrm{mg} / \mathrm{kg}$ to sedate the rabbits and sacrificed them by $\mathrm{CO}_{2}$ inhalation.

2.14. Tissue Harvest. Posteuthanasia, joint surfaces were grossly evaluated. The proximal, tibial, and distal femoral plateaus were removed. Specimens were decalcified with Decalcifier II (Leica Surgipath, Harbourfront Centre, Singapore) for 3-5 days, then placed into $10 \%$ formalin, and cut into four pieces. All four pieces were paraffin-embedded. We performed serial sagittal sections, $4 \mu \mathrm{m}$ in thickness.

2.15. Histological Evaluation. Tissue sections were stained with H\&E (Sigma) to evaluate cellular architecture and 0.1\% Safranin O solution (Sigma) to detect cartilage repair and to demonstrate the matrix's proteoglycan contents. Immunohistochemical staining was performed on additional sections. Specifically, an aggrecan antibody $(1: 200)$, anti-human nuclei antibody $(1: 200)$, and anti-rabbit CD45 (1:200) were used for immunohistochemistry. Reactivity was detected with a diaminobenzidine tetrahydrochloride substrate. Images were captured with a digital camera under a light microscope (Nikon, Tokyo, Japan).

Quantification of aggrecan+ cells in cartilage was performed using 3 fields near injury sites and number of aggrecan + cells among 50 cells counted in each field.

Quantitative evaluation of cartilage repair was performed using cartilage thickness and using the International Cartilage Repair Society (ICRS) scoring system [31]. We randomly selected three slides of the three rabbits and counted the thickness of the destruction site with or without iPSCMSC-chondrocytes transplantation. Subsequently, the mean and standard deviation of the cartilage's thickness was calculated. With the ICRS score, we scored the cartilage from the three rabbits with six categories including the surface, matrix, cell distribution, cell population viability, subchondral bone, and cartilage mineralization. The scores ranged from 0 to 18 .
2.16. Statistical Analysis. All data were expressed as mean and standard deviation. Statistical comparisons of the gene expressions, cartilage's thickness, aggrecan+ cells, and ICRS scores in both groups were performed with nonparametric tests, such as the Mann-Whitney $U$ test. To compare three genes' expression levels, we used ANOVA test with a post hoc analysis with the Bonferroni test. A $p$ value $<0.05$ was considered as statistically significant. All statistical analyses were performed on SPSS (version 22 for Windows; IBM, Inc., New York, NY, USA).

\section{Results}

3.1. Characterization of iPSCs and the Derived Embryoid Body and Xenograft Tumor. The pluripotency of iPSCs was evaluated by demonstrating that iPSCs immunofluorescence staining against NANOG, OCT4, and SOX2 were positive (Figure 1(a)). Of note, immunofluorescence staining of the in vitro differentiated embryoid body showed positive against three germ layer markers including brachyury (mesoderm), tuj-1 (ectoderm), and AFP (endoderm) (Figure 1(b)). RTPCR evaluation of the pluripotent gene expression of iPSCs demonstrated that they could express NANOG, OCT4, and SOX2 (Figure 1(c)). Additionally, RT-PCR also indicated the gene expression of iPSC-derived tumor tissue in three germ layers, demonstrating expressions of GATA4 in endoderm; HAND1 and GATA6 in mesoderm; and TUBB3, $M A P 2$, and GFAP in ectoderm (Figure 1(d)). These germ layers gene expressions except for MAP2 were more increased in xenograft than in the iPSCs (Figure 2). Taken together, iPSCs were pluripotent and could differentiate into three germ layers in vitro and in vivo.

3.2. The Process of MSCs Generation from iPSCs. Undifferentiated iPSCs were plated onto a Matrigel-coating dish on Day 0 . Subsequently, the medium was changed to the MSC culture medium (DMEM $+10 \% \mathrm{FBS}$ ), once every 3 days. On Day 7 (Figure 3(a)), spindle-shaped cells appeared around the colony core (left side of the figure). At one week later, these cells were passaged to an uncoated dish for subsequently enriched cultures (Day 15, Figure 3(b)). When the cells reached $70 \%$ confluence, they were passaged at a ratio of 1:3. On Days 22 (Figure 3(c)) and 29 (Figure 3(d)), most of the cell population was spindle-shaped. Figures $3(\mathrm{e})$ and 3(f) show that gene expression changed from iPSCs to MSCs, indicating the OCT4 was less expressed and CD73 more expressed in MSCs than in iPSCs. Taken together, MSC was differentiated successfully from iPSC after 30 days of differentiation.

\subsection{Characterization of iPSC-Derived MSCs}

3.3.1. Surface Markers. After 30 days of MSCs differentiation process, the surface markers of iPSC-derived MSCs by flow cytometry were positive for CD29, CD44, CD73, CD90, CD105, CD117, and HLA-ABC and negative for CD14, CD19, CD34, CD45, CD56, CD133, and HLA-DR (Figure 4(a)). The surface markers panel was almost the same as bone marrow stem cells (BMSCs) (Figure 4(b)). 

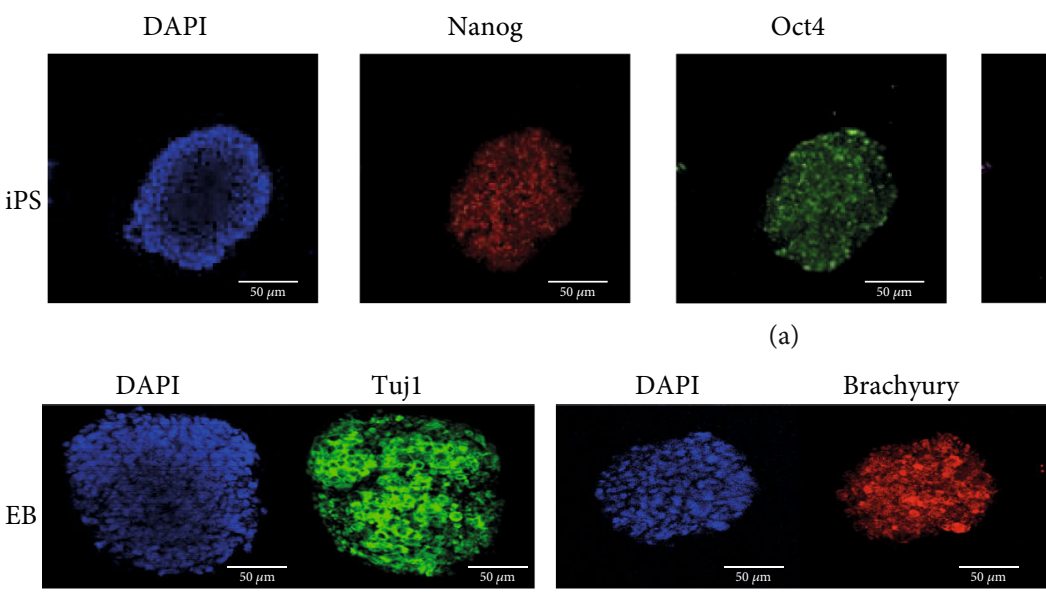

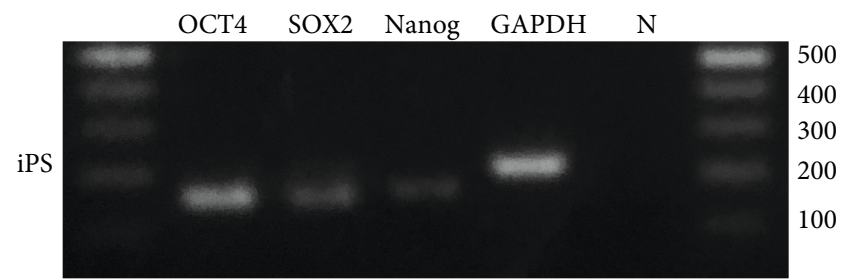

(c) (b)
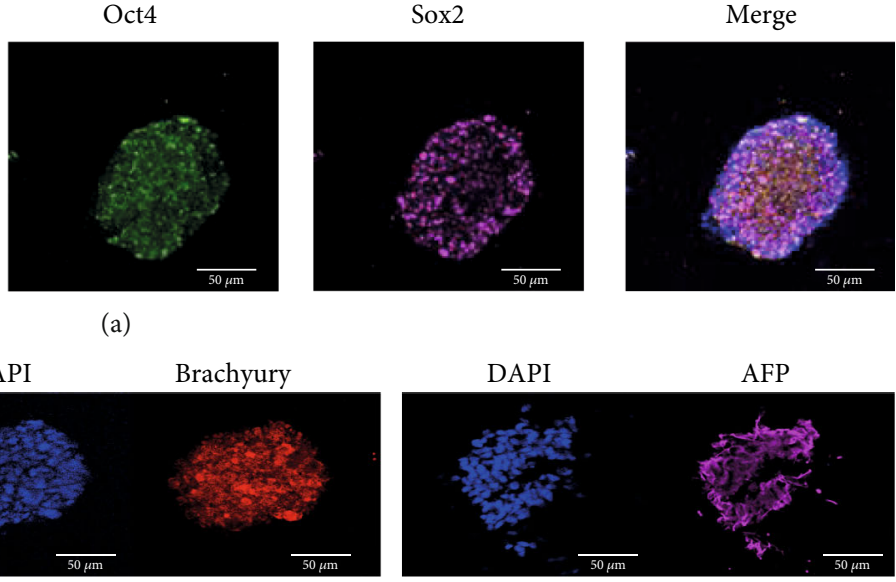

Meso Ecto



(d)

FIGURE 1: Characterization of iPSCs, embryoid body, and teratoma. (a) Counterstaining the nucleus with DAPI as blue color. Immunofluorescence staining against pluripotency markers including NANOG (red), OCT4 (green), and SOX2 (pink). The last figure was a merged picture. Scale bars $=50 \mu \mathrm{m}$. (b) Counterstaining the nucleus with DAPI as blue color. Immunofluorescence staining of embryoid body differentiation against TUJ-1 (ectoderm, green in color), BRACHYURY (mesoderm, red in color), and AFP (endoderm, pink in color). (c) RT-PCR of pluripotency gene expressions of iPSCs. The iPSCs were positive for pluripotency markers including OCT4, SOX2, and NANOG. (d) RT-PCR of gene expression of iPSC-derived teratoma. The teratoma expressed genes of three germ layers, including GATA4 (endoderm), HAND1, GATA6 (mesoderm), TUBB3, MAP2, and GFAP (ectoderm).

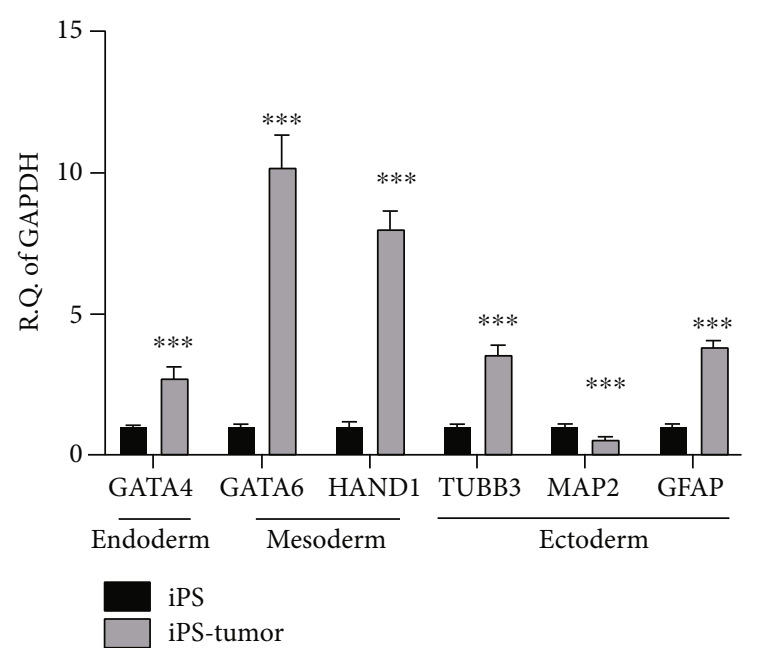

FIGURE 2: Gene expressions of iPSC-derived teratoma compared to iPSC. qPCR showed teratoma expressing more gene expressions of GATA4 (endoderm), HAND1, GATA6 (mesoderm), TUBB3, $M A P 2$, and GFAP (ectoderm) than iPSC. Statistical analyses between both groups were calculated with Mann-Whitney $U$ test. ${ }^{* * *} p<0.001$.
In summary, iPSC-MSC surface marker expressions were as similar as BMSC.

3.4. The Expression of CD24, CD105, and Potassium Channel of iPSC-MSC and BMSC. Regarding the purity of differentiated iPSC-MSC, previous study has shown isolated CD24(-) CD105(+) iPSC-MSC could generate pure MSC and MSC could express different potassium channel patterns [32-35]. Therefore, we compared the CD24, CD105, and potassium channel in both cells. We found that there were 10\% iPSCMSC expressed CD24 and 99.9\% iPSC-MSC expressed CD105, whereas 0.7\% BMSC expressed CD24 and 99.6\% BMSC expressed CD105 (Figure 5(a)). Potassium channel expressions were the same between iPSC-MSC and BMSC (Figures 5(b)-5(d)). Taken together, the expression pattern of iPSC-MSC was almost the same as BMSC except CD24.

3.5. Differentiation of iPSC-Derived MSCs. Next, we checked the differentiation capability of iPSC-derived MSCs. In the adipogenesis of iPSC-MSC, the cells showed positive staining for Oil red, an adipose cell marker (Figure 6(a)). In osteogenesis of iPSC-MSCs, the cells were positive for Alizarin Red, an osteocyte marker (Figure 6(b)). Following chondrogenesis of iPSC- 
DAY 7

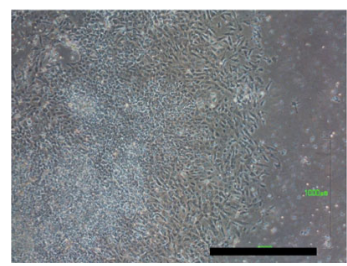

(a)
DAY 15

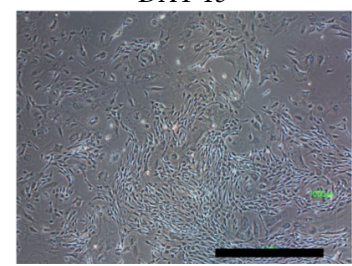

(b)

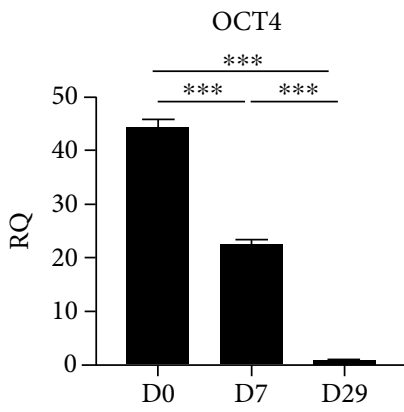

(e)
DAY 22

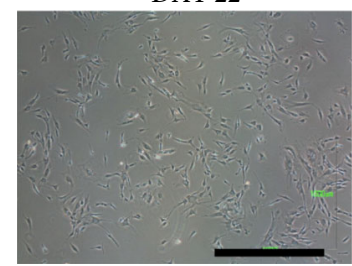

(c)
DAY 29



(d)

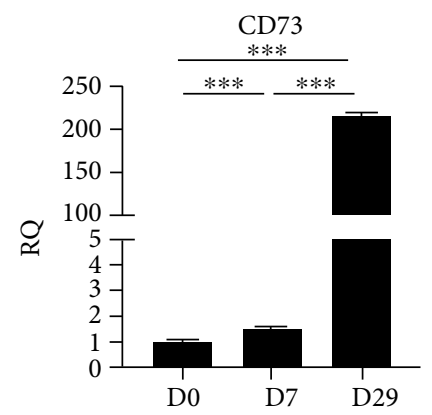

(f)

FIgURE 3: The process from iPSC to mesenchymal stem cell (MSC). (a) The fibroblastic morphology cells (Lt side of the figure) appeared on day 7 after differentiation around the epithelial round cell colony (Rt side of the figure). (b) At day 14, more fibroblast-like cells appeared. (c) At day 21 , around $100 \%$ of cells were fibroblastic appearance cells. (d) On day 29, fully differentiated MSC were noted. Scale bar $=1000 \mu \mathrm{m}$. (e) qPCR showed a decreasing trend of pluripotency gene (OCT4) expression from postdifferentiation day 7 to day 29 . (f) qPCR showed an increasing trend of MSC gene (CD73) expression from postdifferentiation day 7 to day 29. ${ }^{* * *} p<0.001$. We used an ANOVA test with a post hoc analysis with the Bonferroni test.
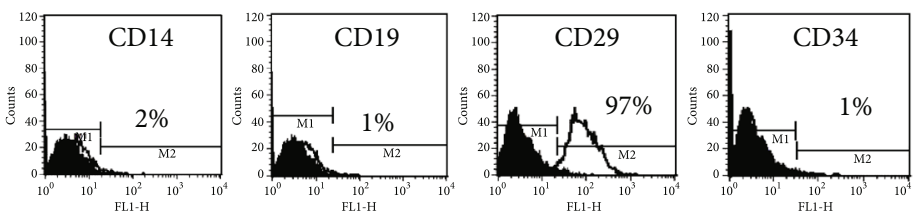

iPSC-MSC
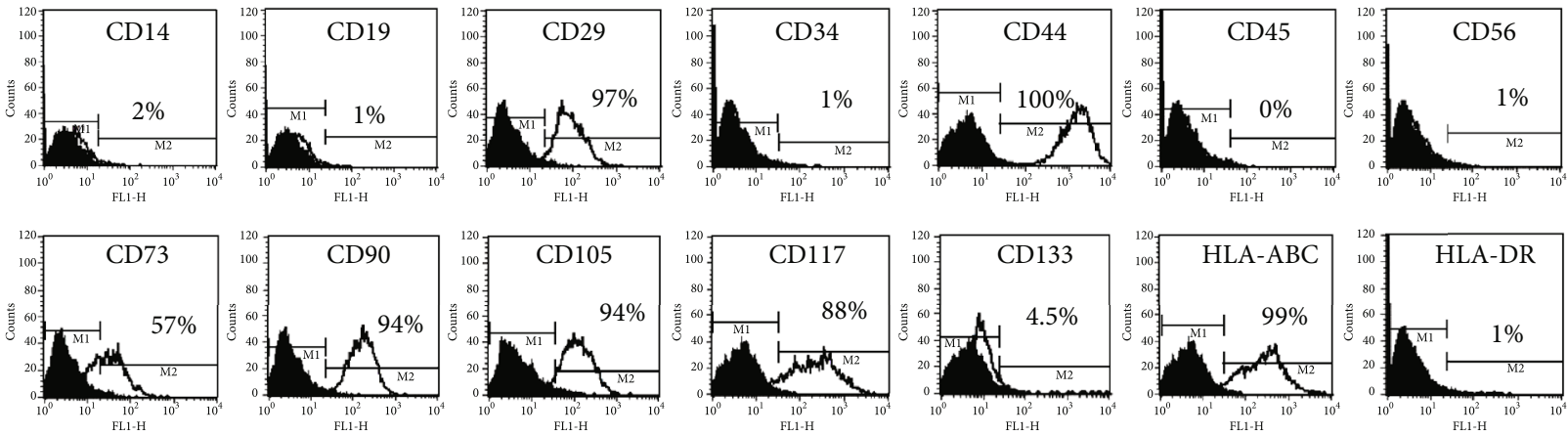

(a) iPSC-MSC
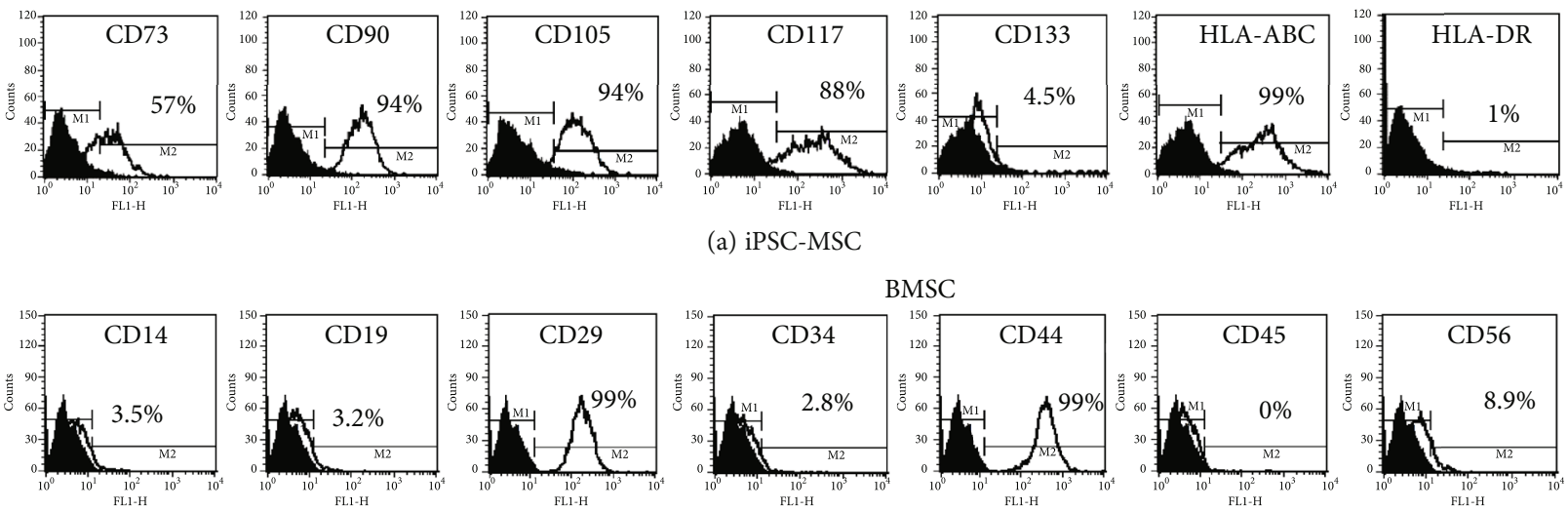

BMSC
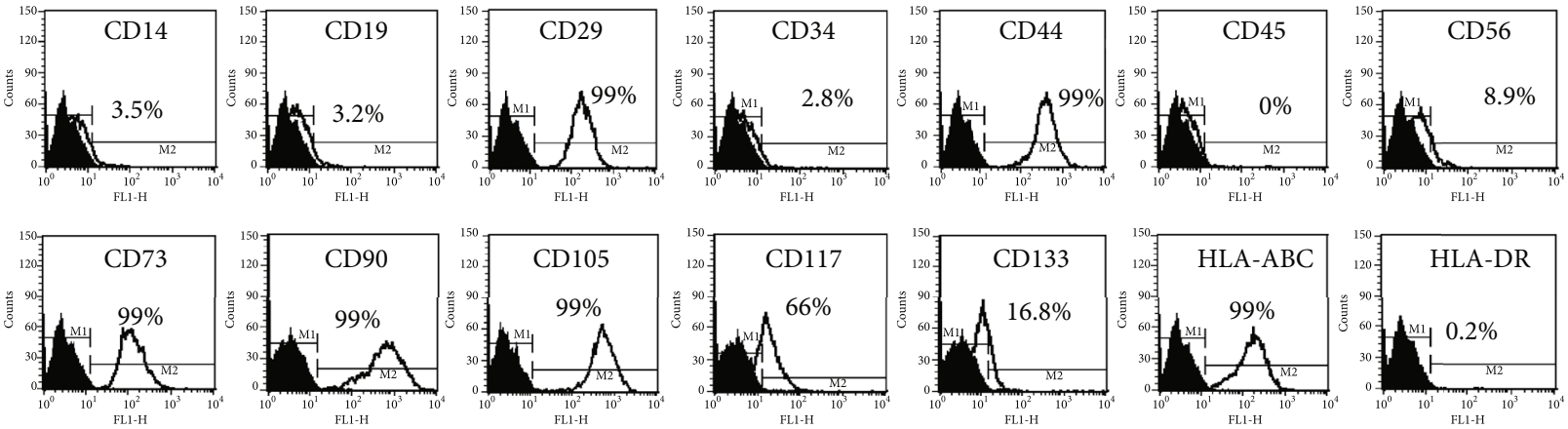

(b) BMSC


FIGURE 4: Flow cytometry of iPSC-MSC and bone marrow stem cells (BMSC). (a) Surface marker expression of iPSC-derived MSCs. They have positive expressions of CD29, CD44, CD73, CD90, CD105, CD117, and HLA-ABC and negative for CD14, CD19, CD34, CD45, CD56, CD133, and HLA-DR. (b) Surface markers expression of BMSC. The expression patterns were similar to iPSC-MSC. 

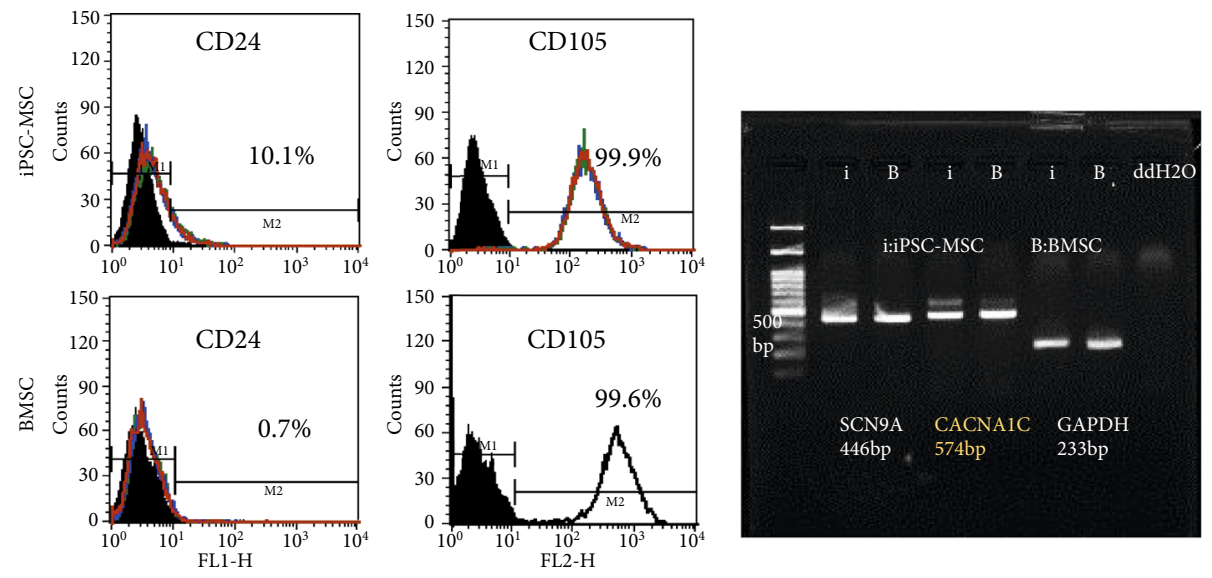

(a)

(b)

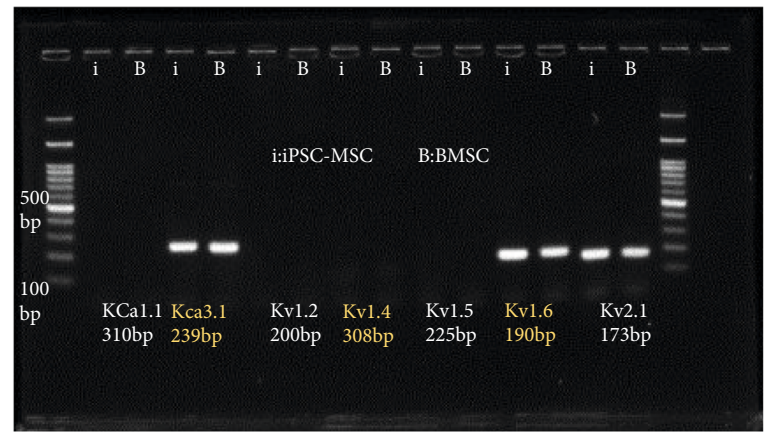

(c)

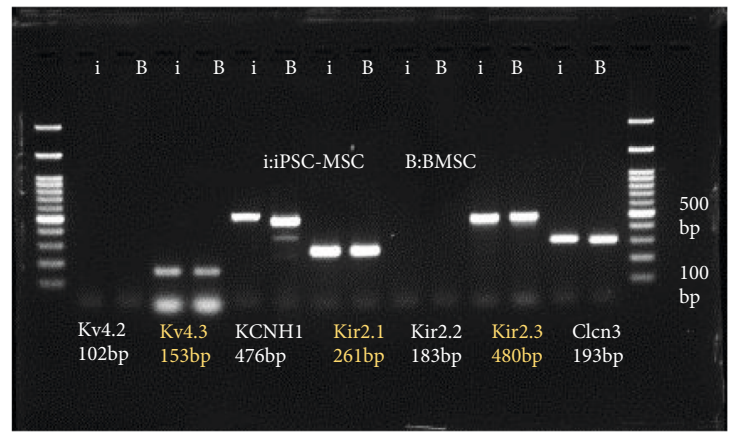

(d)

Figure 5: CD24, CD105, and potassium channel expression in iPSC-MSC and BMSC. (a) CD24 and CD105 expressions in iPSC-MSC and BMSC. CD24 was expressed differently between both cells (10\% in iPSC-MSC and 0.7\% in BMSC). (b-d) RT-PCR showing the potassium channel expressions in iPSC-MSC and BMSC. Both cells expressed potassium channel genes similarly.

derived MSCs for 3 weeks, the cells formed a micromass (Figure 6(c)). H\&E staining of the micromass showed a cartilage-like appearance (Figure 6(d)). The micromass was positive for Safranin $\mathrm{O}$ (sulfated glycosaminoglycans, Figure 6(e)), aggrecan (Figure 6(g)), and type 2 collagen (Figure 6(h)) staining. Figure 6(f) presents negative control of immunohistochemistry.

Quantitative PCR was used to demonstrate the gene expressions of trilineage differentiation of iPSC-MSC. It showed an increased expression of adipocyte-related genes, FABP4 and PPAR $\gamma$ (Figures $7(\mathrm{a})$ and $7(\mathrm{~b})$ ); osteoblastrelated genes, osteopontin $[O P N]$ and alkaline phosphatase [ALPL] (Figures $7(\mathrm{c})$ and $7(\mathrm{~d})$ ); and chondrocyte-related genes, type II collagen and aggrecan (Figures 7(e) and 7(f)) after differentiation. Taken together, iPSC-MSC could proceed trilineage differentiation.

3.6. Microscopic Findings after in vivo Transplantation. After ACLT 8 weeks, we transplanted iPSC-differentiated MSCderived chondrocytes (iPS-MSC-chondrocytes) to repair cartilage defects with OA. After 12 weeks of transplantation, slices of cartilage were evaluated by histology and immunohistochemistry staining. The iPSC-MSC-chondrocytes transplanted cartilage demonstrated more intact histology (Figures 8(a) and 8(b)), positivity for Safranin O (Figures $8(\mathrm{c})$ and $8(\mathrm{~d})$ ), and aggrecan (Figure 9). Figure 9(e) shows the quantification of aggrecan+ cells per 50 cells in the cartilage. There were more aggrecan+ cells noted in the transplanted cartilage.

In a semiquantitative analysis of the sections, the histological assessment of ICRS scores of the three rabbits showed that the repaired tissues in the treated knees were histologically superior to those in the control knees (Figure 10, $n=3, p=0.02$ ).

Taken together, microscopically, we proved iPSC-MSCchondrocytes transplanted cartilages were more intact than control cartilage.

3.7. iPSC-MSC-Chondrocytes Transplantation Decreased Inflammatory Cytokines and Catabolic Matrix Protein Expression. To know the mechanism of how the cartilage defect was repaired by iPSC-MSC-chondrocytes, we performed IHC staining with an antibody against human mitochondria and rabbit CD45, and gene expressions of several inflammatory markers and type 1 and 2 collagen. In $\mathrm{H} \&$ E staining, we found control cartilage showed more destruction of cartilage and less chondrocytes than the treated (Figure 11(a)). There were no rabbit CD45 cells activation in both control and treated cartilage (Figure 11(b)). We did not find human cells integrated into the rabbit cartilage (Figure 11(c)). 


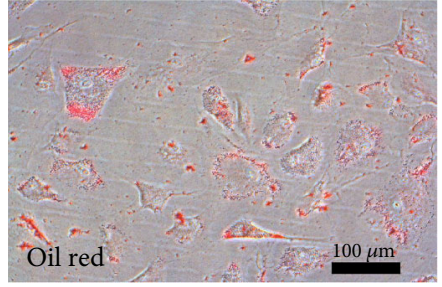

(a)

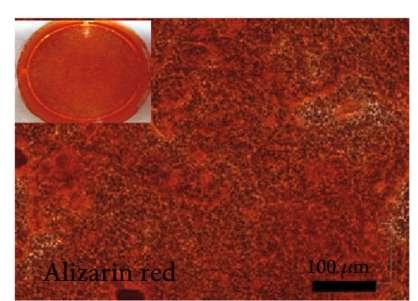

(b)

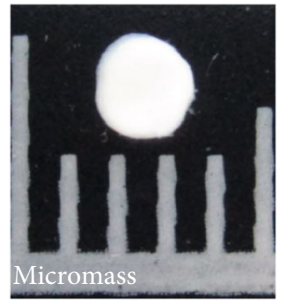

(c)

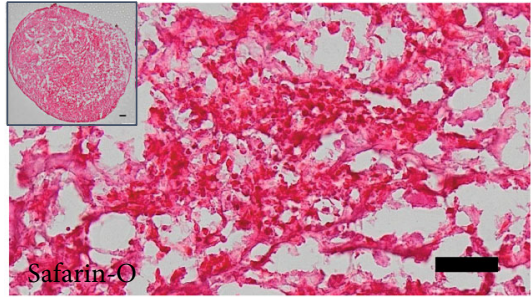

(e)

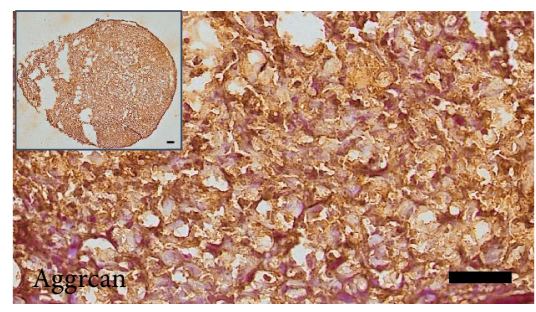

(g)



(d)

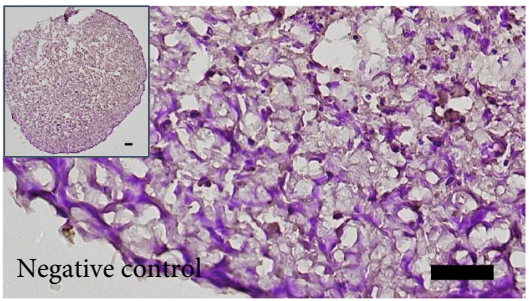

(f)

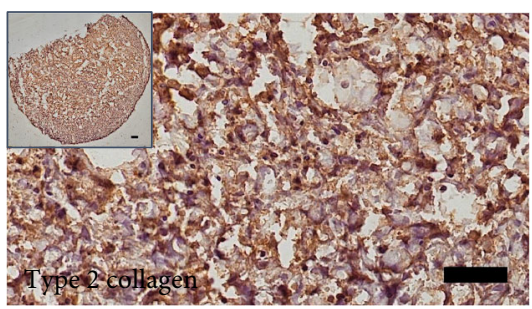

(h)

Figure 6: Trilineage differentiation of iPSC-derived MSCs. (a) After the adipogenesis of iPSC-MSC for 2 weeks, the oil droplets in the cells were staining positive for Oil red. Scale bar $=100 \mu \mathrm{m}$. (b) After the osteogenesis of iPSC-MSC for 2 weeks, the mineral deposition in cells was staining positive for Alizarin Red. Insert picture was Alizarin Red staining of one well of a 6-well plate. Scale bar $=100 \mu \mathrm{m}$. (c) After chondrogenesis of iPSC-derived MSCs for 21 days, the iPSC-MSC formed a cartilage micromass. The picture is $5 \mathrm{~mm}$ in length. (d) The Hematoxylin and Eosin (H \& E) staining of the micromass. (e, g, h) Micromasses were positive for sulfated glycosaminoglycans (Safranin O) (e), aggrecan (g), and type 2 collagen (h). (f) Negative control staining for immunohistochemistry (without the first antibody). Insert pictures in $(\mathrm{d}-\mathrm{h})$ were the picture of the whole micromass section. Scale bar $=100 \mu \mathrm{m}$.

We found inflammatory and catabolic markers, including IL- $1 \beta$, TNF- $\alpha$, and MMP13, decreased expressions after iPS-MSC-chondrocytes transplant (Figures 12(a)-12(c)). However, we did not find the difference of type 2 collagen expression between control and transplanted cartilage (Figure 12(d)). The type 1 collagen expression was also not significantly different between both groups (Figure 12(e)).

Taken together, the mechanism of iPSC-MSCchondrocytes might contribute to their anti-inflammatory and anticatabolic characteristics.

\section{Discussion}

In the present study, we demonstrated that iPSCs can differentiate into MSCs after 30 days, without an embryoid body formation process. The MSCs could successfully differentiate into chondrocytes within 3 weeks. Of note, we found that the differentiated chondrocytes effectively repaired cartilage defects in vivo by anti-inflammatory and anticatabolic mechanisms.

iPSCs offer several advantages over bone marrowderived stem cells (BMSCs) and chondrocytes for cartilage repair [36]. Specifically, iPSC can provide an abundant cell source and a patient-specific in vitro model to study genetic and environmental factors influencing the pathogenesis of OA. Moreover, BMSC and adipose stem cells revealed limited differentiation capability after passage 4 [37]. On the contrary, iPSC can provide an indefinite expansion [38]. The other cell source with an indefinite expansion ability is the embryonic stem cells (ESCs). 


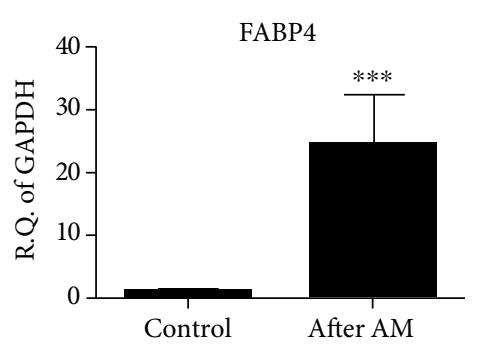

(a)



(d)

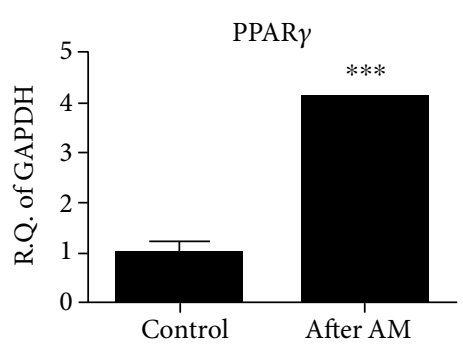

(b)

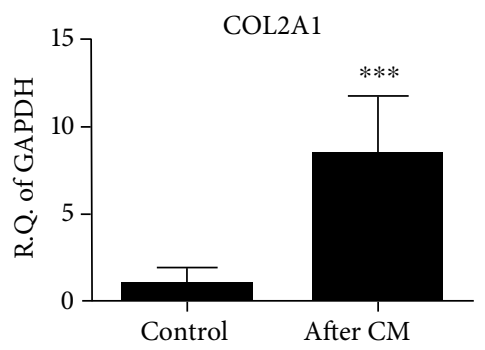

(e)

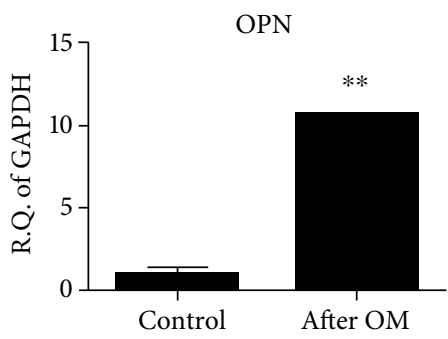

(c)

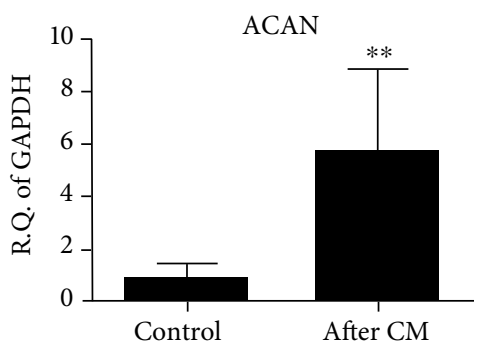

(f)

FIGURE 7: Gene expressions of iPSC-MSC trilineage differentiations. (a-f) qPCR showed an increased expression of (a, b) adipocyte-related genes (FABP4 and PPAR ), (c, d) osteocyte-related genes (osteopontin [OPN] and alkaline phosphatase $[A L P L]$ ), and (e, f) chondrocyterelated genes (type II collagen [COL2A1] and aggrecan $[A C A N]$ ) genes postdifferentiation. AM: adipogenesis medium; OM: osteogenesis medium; CM: chondrogenesis medium. ${ }^{* *} p<0.01,{ }^{* * *} p<0.001$. The experiment was performed thrice. Statistical analyses between both groups were calculated with Mann-Whitney $U$ test.

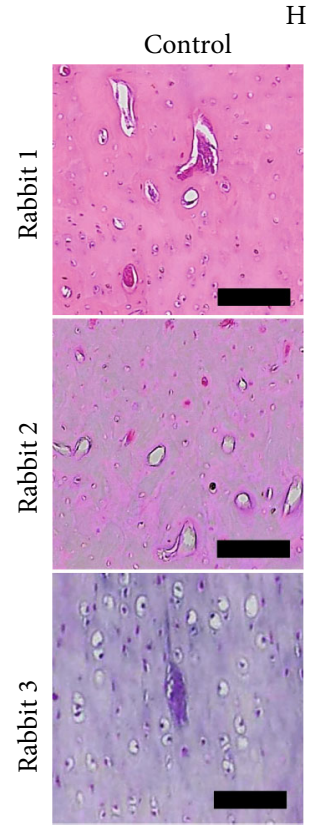

(a)
$H \& E$

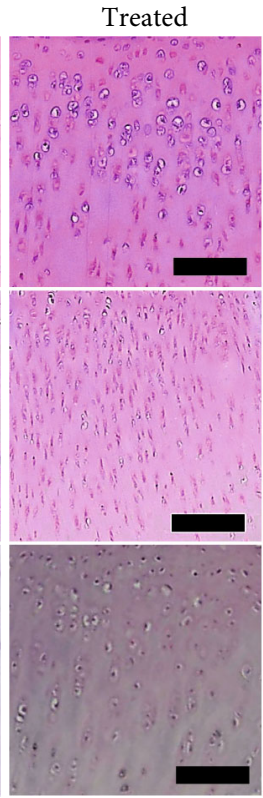

(b)

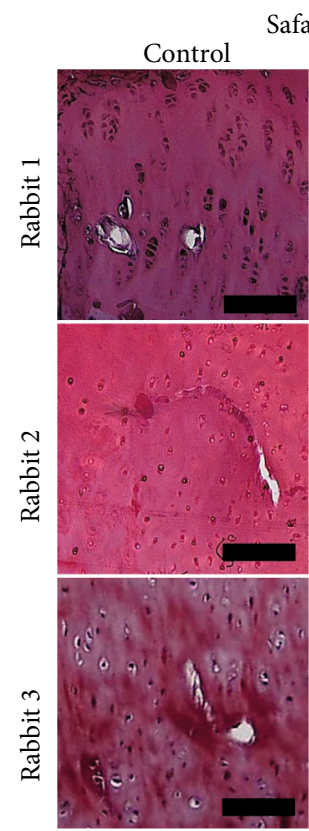

(c)

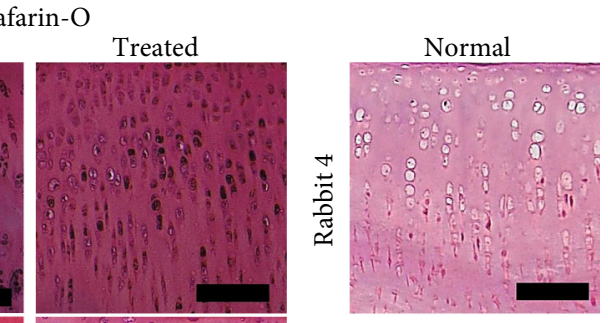

(e)

FIGURE 8: The histology of cartilages with or without iPSC-MSC-chondrocytes transplantation $(n=3)$. (a, b) The H \& E staining of histology of the cartilage of the osteoarthritis (OA) rabbit model without (a) and with cell transplantation (b). (c, d) Safranin O staining of cartilage without (c) or with (d) cells transplantation. (e) Normal rabbit cartilage with Safranin O staining. After iPSC-MSC-chondrocytes transplantation, the morphology and sulfated glycosaminoglycan expressions of transplanted cartilages were more similar to normal cartilage than control cartilage. Scale bar $=100 \mu \mathrm{m}$.

However, the ESCs harbor allogeneic properties, generating immune reactions posttransplantation, limiting their clinical application potential [38]. Compared with other
MSC sources, the generation process is minimally invasive, such as taking skin fibroblasts or blood cells, even from OA suffering elderly population [39]. 


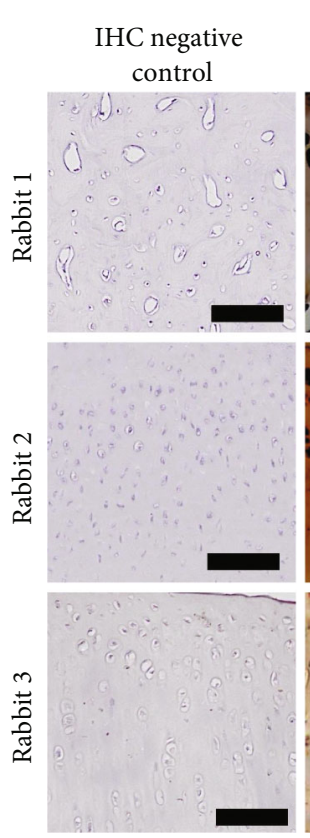

(a)

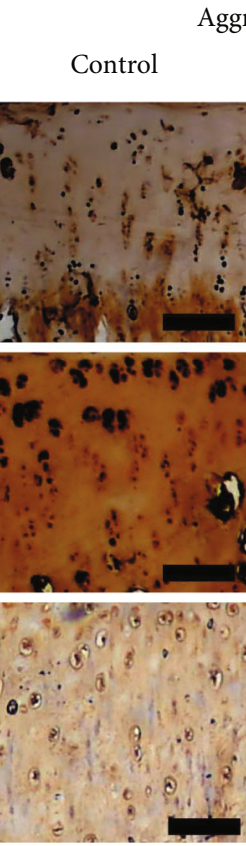

(b)

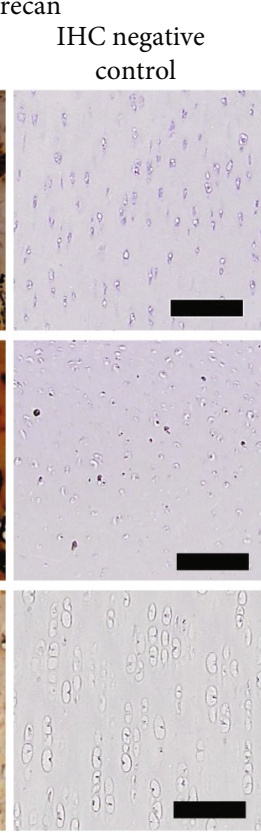

(c)

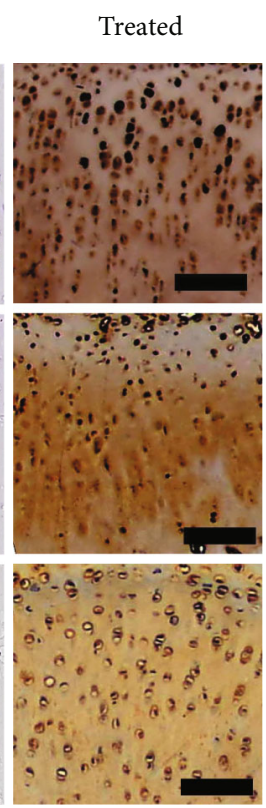

(d)

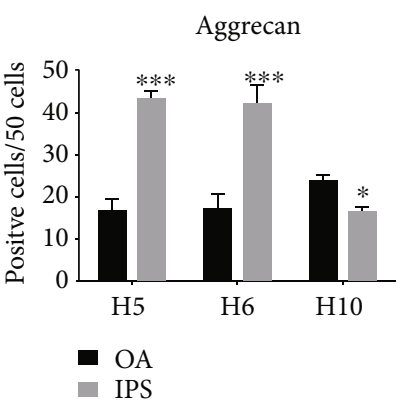

(e)

FIgURE 9: The immunohistochemistry (IHC) in iPSC-MSC-chondrocytes transplant cartilages $(n=3)$. (a, b) The IHC of aggrecan in control cartilage with IHC-negative control (a) and staining cartilage (b). (c, d) IHC of aggrecan in treated cartilage with IHC-negative control (c) and staining cartilage (d). Scale bar $=100 \mu \mathrm{m}$. (e) Quantification of aggrecan + cells in 50 cells. More aggrecan+cells/50 cells were noted in rabbit 1 and $2 .{ }^{*} p<0.5,{ }^{* * *} p<0.01$. Statistical analyses between both groups were calculated with Mann-Whitney $U$ test.

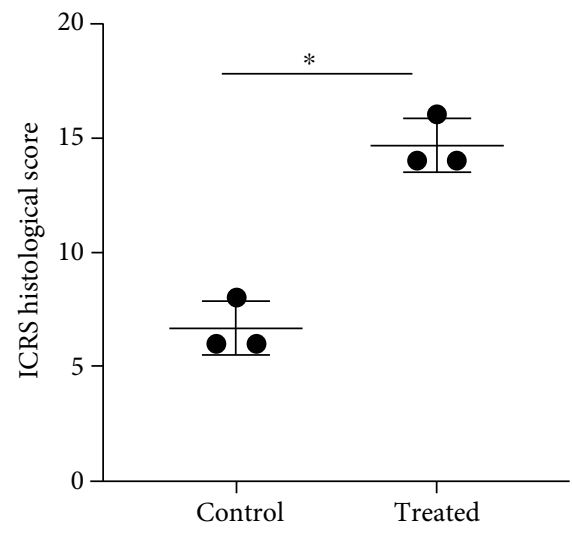

FIgure 10: The International Cartilage Repair Society (ICRS) histological score for evaluating tissue regeneration in osteochondral defects in rabbit articular cartilage at 12 weeks posttreatment. The ICRS histological scores in the treatment group (iPSC-MSC-chondrocytes) were significantly higher than those in the control group $(n=3)$. ${ }^{*} p=0.02$. Statistical analyses between both groups were calculated with Mann-Whitney $U$ test.

The iPSC owned a comparable immunomodulation effect than MSC. Previous studies demonstrated that MSCs have immunosuppressive effects on mitogen-activated immune cells $[40,41]$. The iPSC-MSC is also proved to inhibit mitogen-activated peripheral blood mononuclear cells (PBMC) [42]. The iPSC-MSC owns low tumorigenicity and strong immunomodulatory effects [43]. Moreover, iPSCMSC could induce immune tolerance after transplantation to support long-term survival of graft [44]. Taken together, iPSC-MSC might be a good alternative cell source.

Although iPSC has numerous advantages in cell number, expansion, and differentiation capabilities, the use of iPSC for cartilage repair has been restricted to date, due to the complexity of differentiation protocol and the inefficiency of chondrocytes generation. Currently, four methods are known for the generation of iPSC-derived chondrocytes [45]. The first one is monolayer culture with a defined growth factor [46]; the second one is differentiation into the embryoid body with chondrogenic medium, with TGF- $\beta 3$ cells [47]; the third one is a conditioned medium derived from chondrocytes and added in the culture medium of the embryoid body; and the last one is embryoid body cultured in a chondrocyte condition medium-plus growth factor of TGF- $\beta 3$. Of note, MSCs selected from the embryoid body are costly and laborious, providing low cellular yields [48, 49]. It was concluded that monolayer culture with growth factors and culture with chondrocyte condition medium were the most effective methods [45]. Our study used monolayer culture in which iPSCs differentiated firstly into MSCs, and then differentiated into chondrocytes. This approach was more efficient in chondrogenesis from iPSC than from EB [46].

Other differentiation methods omitted the formation of the embryoid body, including micromass formation of iPSC [50], pellet condition [51], and cultures on a modified dish or feeder layers [52]. However, these methods may have problems such as difficult clinical translation due to the following reasons: influence on cell proliferation by the 


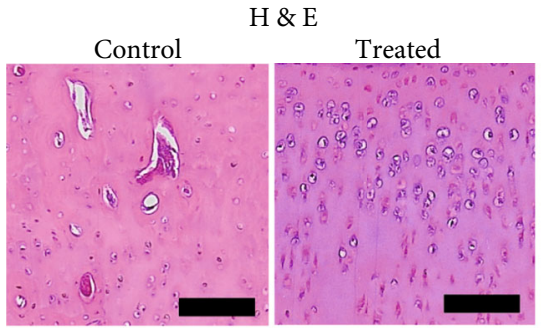

(a)



(b)

Anti-human mitochondria

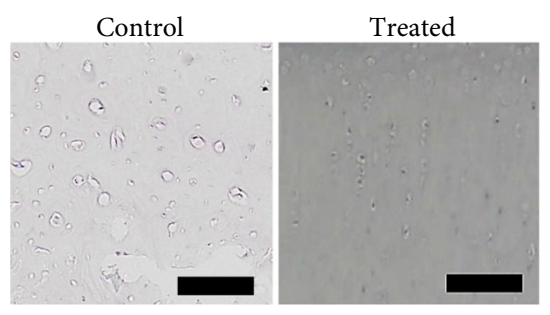

(c)

FIGURE 11: The histology and immunohistochemistry of the iPSC-MSC-chondrocytes transplanted cartilage. (a) H \& E staining of control and treated cartilage. The control cartilage was more destructive than treated cartilage. (b) The IHC of rCD45. There was no staining of rCD45 in both groups. (c) Anti-human mitochondria staining of the cartilage with or without transplanted iPSC-MSC-chondrocytes. There was no staining of anti-human mitochondria in treated cartilage. Scale bar $=100 \mu \mathrm{m}$.



(a)

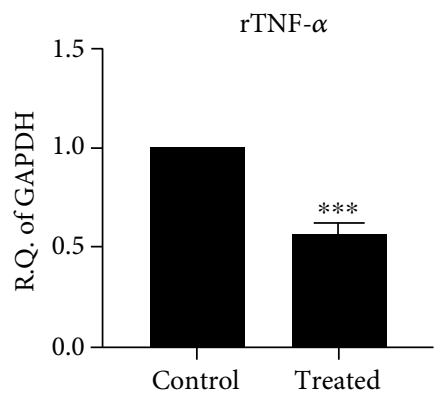

(b)

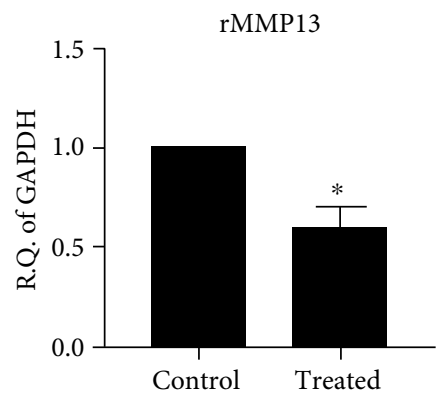

(c)

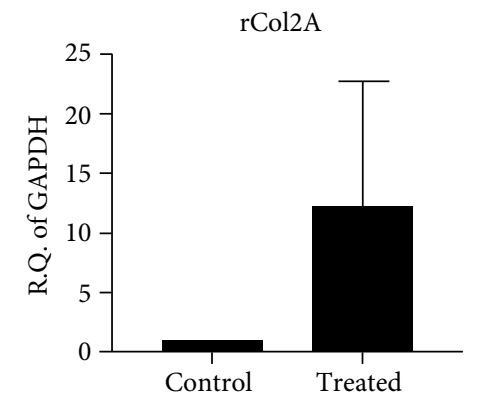

(d)

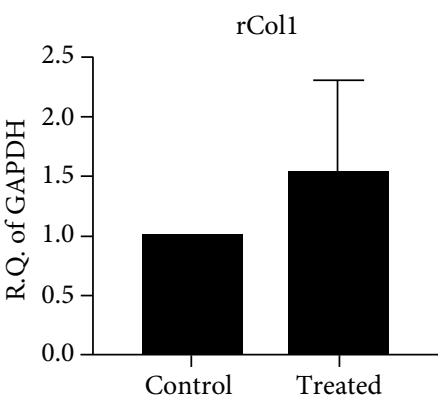

(e)

FIGURE 12: The qRT-PCR of inflammation, catabolic, and cartilage matrix gene expressions in the cartilage with or without transplanted chondrocytes $(n=3)$. (a, b) The inflammatory cytokines, $r I L-1 \beta$ (a) and $r T N F-\alpha$ (b), were illustrated. The inflammatory cytokines decreased expression after cell transplantation. (c) Catabolic matrix gene expression of $r M M P 13$. The expression of the catabolic gene decreased expression after cell transplantation. (d) Gene expression of cartilage matrix of type 2 collagen $[r C O L 2 A]$. The expression of type 2 collagen was not different between control and treated groups. (e) Gene expression of fibrocartilage marker (type 1 collagen $[r C O L 1])$. The expression of type 1 collagen was not different between control and treated groups. ${ }^{*} p<0.05,{ }^{* * *} p<0.001$. Statistical analyses between both groups were calculated with Mann-Whitney $U$ test. 
substrate properties, decrease of related signaling pathways, mixture with feeders, and increased cost [46]. In an additional study, a different method used a TGF- $\beta$ inhibitor to suppress the relative signal in order to enhance MSC conversion [53]. Nevertheless, to date, an in-between tread of the level of epithelialization needed and TGF- $\beta$ inhibitor's safety have not been established. In the present study, we used the easiest way of iPSC-derived chondrocyte via monolayer culture, adding growth factors to facilitate chondrocyte production.

OCT4 expression is abundant in iPSC and reveals the characteristic of pluripotency [54]. Our study showed the iPSC marker and transcription factor OCT4 expression in iPSCs was almost completely lost in the cells after 29 days of transition of iPSCs toward MSCs. The CD73 is one of the classic markers of MSCs [55]. We also demonstrated CD73 was dramatically (200-fold) induced after iPSCs transition toward MSCs.

Regarding purity of iPSC-MSC, previous studies showed they isolated iPSC-MSC CD24(-) CD105(+) cells to generate MSC population [32-34]. These cells could propagate more than 100 passages and perform trilineage differentiations. Besides, human iPSC-MSCs express potassium channel Kir2.2 and Kir2.3 but BMSC do not [35]. Nevertheless, in our study, we found the iPSC-MSC was CD24 low expression (10\%) and CD105 (+). The potassium channel expressions between both kinds of cells were the same, such as Kir2.2 and Kir2.3. Therefore, the purity of iPSC-MSC may be proved.

Prolonged cell culture may induce chondrocytes undergoing hypertrophic changes (i.e., expression of the type $\mathrm{X}$ collagen gene (COL10A1) [56]. In addition, the lack of specific markers as chondrocyte identity makes it difficult to prospectively isolate chondrocytes. Recent fate-mapping studies in the mouse show that chondrocyte-like cells are derived from early notochord-like nucleus pulposus cells (NP cells) [57]. The efforts to derive notochord-like NP cells for chondrocyte differentiation have been also reported recently [58]. The advantages of NP cell-derived chondrocytes are that NP cells are the proceeding cells of the chondrocytes and could be easily identified. The disadvantages of NP cell-derived chondrocytes are needed for one more step of differentiation than iPSC-MSC.

OA is caused by a chronic inflammatory process. Chondrolysis may be caused by increased inflammatory cytokines such as IL- $1 \beta$, TNF- $\alpha$, and catabolic matrix protein MMP13 in the joint [59]. In addition, IL- $1 \beta$ could also inhibit the production of cartilage matrix components such as type 2 collagen and aggrecan [60]. It might be possible that the antiinflammation effects were from iPSC-MSCs instead of iPSC-MSC-chondrocytes. Recent studies also indicated mitochondrial transfer of iPSC-MSC is another putative mechanism to reduce inflammation and change metabolic status of injured cells/tissues [61-63]. In our study, we found that transplanted iPSC-MSC-chondrocytes could inhibit these inflammatory cytokines and catabolic matrix protein.

During iPSC differentiation to MSC (iPSC-MSC), gene expressions of characteristic surface markers of MSC, including CD44, CD73, and CD105, were upregulated [64]. How- ever, the immune modulation function of iPSC-MSC was not as well as genuine MSC [64]. The previous study showed that iPSC-MSC showed rejuvenation from methylation associated with age and senescence compared to genuine MSC [64]. It means iPSC-MSC owns differentiation and immunomodulation advantages of MSC but not owns age- and senescence-associated disadvantages. In our study, we could see the surface marker expressions and differentiation capability of iPSC-MSC were the same as genuine MSC.

The mechanisms of stem cells repair the destroyed cartilage are contributed by stem cell integration and differentiation, immunomodulation, or by the paracrine factors and exosome production [65]. One previous study showed human iPSC, transplanted or cotransplanted with alginate into defected cartilage, could be integrated into defected cartilage and repair [66]. The other study revealed that MSC owned immunomodulation and anti-inflammatory effects to repair the cartilage defect [67]. One review also pointed out that autologous chondrocyte implantation (ACI) is the most established clinical treatment currently [68]. However, there are several disadvantages of ACI including the complexity and cost of surgical procedures, the biological response of the flap, and the complexity of the in vitro expansion of chondrocytes [69]. In our study, we could provide several advantages over ACI. Firstly, the cell numbers are unlimited for iPSC, and the procedures of culturing fibroblast and iPSC are not complex. Secondly, we found chondrocytes differentiated from iPSC-MSC could repair the defective cartilage through the anti-inflammation effect. The chondrocytes would not integrate into defective cartilage but decreased several inflammatory markers such as IL- $1 \beta$, TNF- $\alpha$, and MMP13. To our knowledge, these findings were not reported before. Thirdly, the methylation status of iPSC-MSC was different from the MSC. The iPSCMSC is rejuvenated.

Some limitations can be identified in the present study. First, FBS was used as a nutrient in the culture medium. This could increase the hazard of cell transformation and prion contamination [70]. In the future, for clinical application, a serum replacement could decrease the safety concern [71]. Second, iPSC could undergo teratoma formation. However, here, we performed a terminal differentiation, like chondrocyte differentiation. Therefore, there was a very small chance of teratoma formation. Of note, we did not observe teratoma formation in our experiments. Furthermore, we used the Sendai virus to transfect four factors. The virus is expected to eliminate after 10-13 passages in culture, decreasing the chances of pluripotency factors' reactivation [72]. We did not see the iPSC-MSC-chondrocytes integration after 12 weeks of transplantation. The cells may die after such a long period of time. A short time period of the experiment to see if iPSC-MSC-chondrocytes integration is needed in the future.

\section{Conclusion}

In conclusion, the present study shows the iPSCs we derived could directly differentiate into MSCs and subsequently into chondrocytes, in the absence of embryoid body formation. These iPSC-MSC-chondrocytes might effectively 
repair cartilage defects through anti-inflammatory and anticatabolic mechanisms. Thus, the iPSCs we derived might represent a feasible source of cell therapy for OAdamaged cartilage.

\section{Abbreviations}

ACL: Anterior cruciate ligament

ACLT: Anterior cruciate ligament transection

FBS: Fetal bovine serum

H\&E: Hematoxylin and Eosin

MSC: Mesenchymal stem cell

PRP: Platelet-rich plasma.

\section{Data Availability}

The original data can ask the corresponding author.

\section{Conflicts of Interest}

The authors declare no conflict of interest.

\section{Authors' Contributions}

DCD designed the experiment; DCD, KCW, and YHC performed experiments; DCD, KCW, and YHC analyzed the data; DCD, KCW, and YHC wrote the paper and final approval. YuHsun Chang and Kun-Chi Wu equal contribution.

\section{Acknowledgments}

The authors thank the core facilities provided by the Advanced Instrumentation Center of the Department of Medical Research, Hualien Tzu Chi Hospital, Buddhist Tzu Chi Medical Foundation. The work was supported by the funds of the Buddhist Tzu Chi Medical Foundation and Intramural grants of Hualien Tzu Chi Hospital (TCMMP 104-05, TCRD 109-61, 109-62).

\section{References}

[1] I. Haq, E. Murphy, and J. Dacre, "Osteoarthritis," Postgraduate Medical Journal, vol. 79, no. 933, pp. 377-383, 2003.

[2] T. Hayami, Y. Zhuo, G. A. Wesolowski, M. Pickarski, and L. T. Duong, "Inhibition of cathepsin $\mathrm{K}$ reduces cartilage degeneration in the anterior cruciate ligament transection rabbit and murine models of osteoarthritis," Bone, vol. 50, no. 6, pp. 1250-1259, 2012.

[3] S. B. Abramson and M. Attur, "Developments in the scientific understanding of osteoarthritis," Arthritis Research \& Therapy, vol. 11, no. 3, p. 227, 2009.

[4] Y. Zhang and J. M. Jordan, "Epidemiology of osteoarthritis," Clinics in Geriatric Medicine, vol. 26, no. 3, pp. 355-369, 2010.

[5] J. C. Mora, R. Przkora, and Y. Cruz-Almeida, "Knee osteoarthritis: pathophysiology and current treatment modalities," Journal of Pain Research, vol. Volume 11, pp. 2189-2196, 2018.

[6] W. H. Robinson, C. M. Lepus, Q. Wang et al., "Low-grade inflammation as a key mediator of the pathogenesis of osteoarthritis," Nature Reviews Rheumatology, vol. 12, no. 10, pp. 580-592, 2016.
[7] E. Ayhan, H. Kesmezacar, and I. Akgun, "Intraarticular injections (corticosteroid, hyaluronic acid, platelet rich plasma) for the knee osteoarthritis," World Journal of Orthopedics, vol. 5, no. 3, pp. 351-361, 2014.

[8] M. M. Richards, J. S. Maxwell, L. Weng, M. G. Angelos, and J. Golzarian, "Intra-articular treatment of knee osteoarthritis: from anti-inflammatories to products of regenerative medicine," The Physician and Sportsmedicine, vol. 44, no. 2, pp. 101-108, 2016.

[9] P. Wehling, C. Evans, J. Wehling, and W. Maixner, "Effectiveness of intra-articular therapies in osteoarthritis: a literature review," Therapeutic Advances in Musculoskeletal Disease, vol. 9, no. 8, pp. 183-196, 2017.

[10] M. Shahid and R. Kundra, "Platelet-rich plasma (PRP) for knee disorders," EFORT Open Reviews, vol. 2, no. 2, pp. 28 34, 2017.

[11] Y.-H. Chang, H.-W. Liu, K.-C. Wu, and D.-C. Ding, "Mesenchymal stem cells and their clinical applications in osteoarthritis," Cell Transplantation, vol. 25, no. 5, pp. 937-950, 2016.

[12] D.-C. Ding, K.-C. Wu, H.-L. Chou, W.-T. Hung, H.-W. Liu, and T.-Y. Chu, "Human infrapatellar fat pad-derived stromal cells have more potent differentiation capacity than other mesenchymal cells and can be enhanced by hyaluronan," Cell Transplantation, vol. 24, no. 7, pp. 1221-1232, 2015.

[13] R. Mafi, S. Hindocha, P. Mafi, M. Griffin, and W. S. Khan, "Sources of adult mesenchymal stem cells applicable for musculoskeletal applications - a systematic review of the literature," The Open Orthopaedics Journal, vol. 5, no. 1, pp. 242248, 2011.

[14] K. Takahashi and S. Yamanaka, "Induction of pluripotent stem cells from mouse embryonic and adult fibroblast cultures by defined factors," Cell, vol. 126, no. 4, pp. 663-676, 2006.

[15] J. Yu, M. A. Vodyanik, K. Smuga-Otto et al., "Induced pluripotent stem cell lines derived from human somatic cells," Science, vol. 318, no. 5858, pp. 1917-1920, 2007.

[16] M. Mandai, A. Watanabe, Y. Kurimoto et al., "Autologous induced stem-cell-derived retinal cells for macular degeneration," The New England Journal of Medicine, vol. 376, no. 11, pp. 1038-1046, 2017.

[17] T. Teramura, Y. Onodera, T. Mihara, Y. Hosoi, C. Hamanishi, and K. Fukuda, "Induction of mesenchymal progenitor cells with chondrogenic property from mouse-induced pluripotent stem cells," Cellular Reprogramming, vol. 12, no. 3, pp. 249261, 2010.

[18] N. Koyama, M. Miura, K. Nakao et al., "Human induced pluripotent stem cells differentiated into chondrogenic lineage via generation of mesenchymal progenitor cells," Stem Cells and Development, vol. 22, no. 1, pp. 102-113, 2013.

[19] Y. Zhu, X. Wu, Y. Liang et al., "Repair of cartilage defects in osteoarthritis rats with induced pluripotent stem cell derived chondrocytes," BMC Biotechnology, vol. 16, no. 1, p. 78, 2016.

[20] T. Kuroda, S. Yasuda, S. Kusakawa et al., "Highly sensitive in vitro methods for detection of residual undifferentiated cells in retinal pigment epithelial cells derived from human iPS cells," PLoS One, vol. 7, no. 5, article e37342, 2012.

[21] K. Matsuura, F. Kodama, K. Sugiyama, T. Shimizu, N. Hagiwara, and T. Okano, "Elimination of remaining undifferentiated induced pluripotent stem cells in the process of human cardiac cell sheet fabrication using a methionine-free culture condition," Tissue Engineering Part C, Methods, vol. 21, no. 3, pp. 330-338, 2015. 
[22] A. Banito and J. Gil, "Induced pluripotent stem cells and senescence: learning the biology to improve the technology," EMBO Reports, vol. 11, no. 5, pp. 353-359, 2010.

[23] I.-H. Park, R. Zhao, J. A. West et al., "Reprogramming of human somatic cells to pluripotency with defined factors," Nature, vol. 451, no. 7175, pp. 141-146, 2008.

[24] A. Banito, S. T. Rashid, J. C. Acosta et al., "Senescence impairs successful reprogramming to pluripotent stem cells," Genes \& Development, vol. 23, no. 18, pp. 2134-2139, 2009.

[25] R. Shrestha, Y.-T. Wen, D.-C. Ding, and R.-K. Tsai, "Aberrant hiPSCs-derived from human keratinocytes differentiates into $3 \mathrm{D}$ retinal organoids that acquire mature photoreceptors," Cell, vol. 8, no. 1, p. 36, 2019.

[26] J. Beers, D. R. Gulbranson, N. George et al., "Passaging and colony expansion of human pluripotent stem cells by enzyme-free dissociation in chemically defined culture conditions," Nature Protocols, vol. 7, no. 11, pp. 2029-2040, 2012.

[27] J. H. Lee, E. J. Lee, C. H. Lee, J. H. Park, J. Y. Han, and J. M. Lim, "Requirement of leukemia inhibitory factor for establishing and maintaining embryonic stem cells in mice," Fertility and Sterility, vol. 92, no. 3, pp. 1133-1140, 2009.

[28] K. Hynes, D. Menicanin, K. Mrozik, S. Gronthos, and P. M. Bartold, "Generation of functional mesenchymal stem cells from different induced pluripotent stem cell lines," Stem Cells and Development, vol. 23, no. 10, pp. 1084-1096, 2014.

[29] K. J. Livak and T. D. Schmittgen, "Analysis of relative gene expression data using real-time quantitative PCR and the 2(Delta Delta C (T)) method," Methods, vol. 25, no. 4, pp. 402408, 2001.

[30] T. Hayami, M. Pickarski, G. A. Wesolowski et al., "The role of subchondral bone remodeling in osteoarthritis: reduction of cartilage degeneration and prevention of osteophyte formation by alendronate in the rat anterior cruciate ligament transection model," Arthritis and Rheumatism, vol. 50, no. 4, pp. 11931206, 2004.

[31] P. Mainil-Varlet, T. Aigner, M. Brittberg et al., "Histological assessment of cartilage repair: a report by the Histology Endpoint Committee of the International Cartilage Repair Society (ICRS)," The Journal of Bone and Joint Surgery. American Volume, vol. 85, Supplement 2, pp. 45-57, 2003.

[32] Q. Lian, Y. Zhang, J. Zhang et al., "Functional mesenchymal stem cells derived from human induced pluripotent stem cells attenuate limb ischemia in mice," Circulation, vol. 121, no. 9, pp. 1113-1123, 2010.

[33] Q. Lian, E. Lye, K. Suan Yeo et al., "Derivation of clinically compliant MSCs from CD105+, CD24- differentiated human ESCs," Stem Cells, vol. 25, no. 2, pp. 425-436, 2007.

[34] Q. Lian, Y. Zhang, X. Liang, F. Gao, and H.-F. Tse, "Directed differentiation of human-induced pluripotent stem cells to mesenchymal stem cells," Methods in Molecular Biology, vol. 1416, pp. 289-298, 2016.

[35] J. Zhang, Y.-C. Chan, J. C.-Y. Ho, C.-W. Siu, Q. Lian, and H.F. Tse, "Regulation of cell proliferation of human induced pluripotent stem cell-derived mesenchymal stem cells via ether-àgo-go 1 (hEAG1) potassium channel," American Journal of Physiology-Cell Physiology, vol. 303, no. 2, pp. C115-C125, 2012.

[36] B. O. Diekman, N. Christoforou, V. P. Willard et al., "Cartilage tissue engineering using differentiated and purified induced pluripotent stem cells," Proceedings of the National Academy of Sciences of the United States of America, vol. 109, no. 47, pp. 19172-19177, 2012.
[37] L. A. Solchaga, K. Penick, V. M. Goldberg, A. I. Caplan, and J. F. Welter, "Fibroblast growth factor-2 enhances proliferation and delays loss of chondrogenic potential in human adult bone-marrow-derived mesenchymal stem cells," Tissue Engineering. Part A, vol. 16, no. 3, pp. 1009-1019, 2010.

[38] Y. Jung, G. Bauer, and J. A. Nolta, “Concise review: induced pluripotent stem cell-derived mesenchymal stem cells: progress toward safe clinical products," Stem Cells, vol. 30, no. 1, pp. 42-47, 2012.

[39] Y. Wei, W. Zeng, R. Wan et al., "Chondrogenic differentiation of induced pluripotent stem cells from osteoarthritic chondrocytes in alginate matrix," European Cells \& Materials, vol. 23, pp. 1-12, 2012.

[40] M. E. Castro-Manrreza, H. Mayani, A. Monroy-García et al., "Human mesenchymal stromal cells from adult and neonatal sources: a comparative in vitro analysis of their immunosuppressive properties against T cells," Stem Cells and Development, vol. 23, no. 11, pp. 1217-1232, 2014.

[41] Z. Yan, Y. Zhuansun, G. Liu, R. Chen, J. Li, and P. Ran, "Mesenchymal stem cells suppress $\mathrm{T}$ cells by inducing apoptosis and through PD-1/B7-H1 interactions," Immunology Letters, vol. 162, no. 1, pp. 248-255, 2014.

[42] Q. L. Fu, Y. Y. Chow, S. J. Sun et al., "Mesenchymal stem cells derived from human induced pluripotent stem cells modulate T-cell phenotypes in allergic rhinitis," Allergy, vol. 67, no. 10, pp. 1215-1222, 2012.

[43] L.-T. Wang, S.-S. Jiang, C.-H. Ting et al., "Differentiation of mesenchymal stem cells from human induced pluripotent stem cells results in downregulation of c-Myc and DNA replication pathways with immunomodulation toward CD4 and CD8 cells," Stem Cells, vol. 36, no. 6, pp. 903-914, 2018.

[44] M. A. Khan, F. Alanazi, H. A. Ahmed et al., "iPSC-derived MSC therapy induces immune tolerance and supports longterm graft survival in mouse orthotopic tracheal transplants," Stem Cell Research \& Therapy, vol. 10, no. 1, p. 290, 2019.

[45] W. M. Suchorska, E. Augustyniak, M. Richter, and T. Trzeciak, "Comparison of four protocols to generate chondrocyte-like cells from human induced pluripotent stem cells (hiPSCs)," Stem Cell Reviews, vol. 13, no. 2, pp. 299-308, 2017.

[46] H. Nejadnik, S. Diecke, O. D. Lenkov et al., "Improved approach for chondrogenic differentiation of human induced pluripotent stem cells," Stem Cell Reviews, vol. 11, no. 2, pp. 242-253, 2015.

[47] W. S. Toh, E. H. Lee, M. Richards, and T. Cao, "In vitro derivation of chondrogenic cells from human embryonic stem cells," Methods in Molecular Biology, vol. 584, pp. 317-331, 2009.

[48] D. J. Kahler, F. S. Ahmad, A. Ritz et al., "Improved methods for reprogramming human dermal fibroblasts using fluorescence activated cell sorting," PLoS One, vol. 8, no. 3, article e59867, 2013.

[49] S. B. Bahrami, M. Veiseh, and N. J. Boudreau, "Isolation and expansion of endothelial progenitor cells derived from mouse embryonic stem cells," Methods in Molecular Biology, vol. 916, pp. 81-96, 2012.

[50] B. Saitta, J. Passarini, D. Sareen et al., "Patient-derived skeletal dysplasia induced pluripotent stem cells display abnormal chondrogenic marker expression and regulation by BMP2 and TGF $\beta 1$," Stem Cells and Development, vol. 23, no. 13, pp. 1464-1478, 2014.

[51] M. D. Phillips, S. A. Kuznetsov, N. Cherman et al., "Directed differentiation of human induced pluripotent stem cells 
toward bone and cartilage: in vitro versus in vivo assays," Stem Cells Translational Medicine, vol. 3, no. 7, pp. 867-878, 2014.

[52] L. G. Villa-Diaz, S. E. Brown, Y. Liu et al., "Derivation of mesenchymal stem cells from human induced pluripotent stem cells cultured on synthetic substrates," Stem Cells, vol. 30, no. 6, pp. 1174-1181, 2012.

[53] Y. S. Chen, R. A. Pelekanos, R. L. Ellis, R. Horne, E. J. Wolvetang, and N. M. Fisk, "Small molecule mesengenic induction of human induced pluripotent stem cells to generate mesenchymal stem/stromal cells," Stem Cells Translational Medicine, vol. 1, no. 2, pp. 83-95, 2012.

[54] G. Shi and Y. Jin, "Role of Oct 4 in maintaining and regaining stem cell pluripotency," Stem Cell Research \& Therapy, vol. 1, no. 5, p. 39, 2010.

[55] M. Breitbach, K. Kimura, T. C. Luis et al., "In vivo labeling by CD73 marks multipotent stromal cells and highlights endothelial heterogeneity in the bone marrow niche," Cell Stem Cell, vol. 22, no. 2, pp. 262-76.e7, 2018.

[56] K. Pelttari, A. Winter, E. Steck et al., "Premature induction of hypertrophy during in vitro chondrogenesis of human mesenchymal stem cells correlates with calcification and vascular invasion after ectopic transplantation in SCID mice," Arthritis and Rheumatism, vol. 54, no. 10, pp. 3254-3266, 2006.

[57] S. Mohanty, R. Pinelli, P. Pricop, T. J. Albert, and C. L. Dahia, "Chondrocyte-like nested cells in the aged intervertebral disc are late-stage nucleus pulposus cells," Aging Cell, vol. 18, no. 5, p. e13006, 2019.

[58] Y. Zhang, Z. Zhang, P. Chen et al., "Directed differentiation of notochord-like and nucleus pulposus-like cells using human pluripotent stem cells," Cell Reports, vol. 30, no. 8, pp. 2791806.e5, 2020.

[59] J. Sokolove and C. M. Lepus, "Role of inflammation in the pathogenesis of osteoarthritis: latest findings and interpretations," Therapeutic Advances in Musculoskeletal Disease, vol. 5, no. 2, pp. 77-94, 2013.

[60] M. Maldonado and J. Nam, "The role of changes in extracellular matrix of cartilage in the presence of inflammation on the pathology of osteoarthritis," BioMed Research International, vol. 2013, Article ID 284873, 10 pages, 2013.

[61] D. Jiang, G. Xiong, H. Feng et al., "Donation of mitochondria by iPSC-derived mesenchymal stem cells protects retinal ganglion cells against mitochondrial complex I defect-induced degeneration," Theranostics, vol. 9, no. 8, pp. 2395-2410, 2019.

[62] Y. Zhang, Z. Yu, D. Jiang et al., "iPSC-MSCs with high intrinsic MIRO1 and sensitivity to TNF- $\alpha$ yield efficacious mitochondrial transfer to rescue anthracycline-induced cardiomyopathy," Stem Cell Reports, vol. 7, no. 4, pp. 749-763, 2016.

[63] A. C. Court, A. le-Gatt, P. Luz-Crawford et al., "Mitochondrial transfer from MSCs to T cells induces Treg differentiation and restricts inflammatory response," EMBO Reports, vol. 21, no. 2, article e48052, 2020.

[64] J. Frobel, H. Hemeda, M. Lenz et al., "Epigenetic rejuvenation of mesenchymal stromal cells derived from induced pluripotent stem cells," Stem Cell Reports, vol. 3, no. 3, pp. 414-422, 2014.

[65] M. L. Monaco, G. Merckx, J. Ratajczak et al., "Stem cells for cartilage repair: preclinical studies and insights in translational animal models and outcome measures," Stem Cells International, vol. 2018, Article ID 9079538, 22 pages, 2018.
[66] J.-Y. Ko, K.-I. Kim, S. Park, and G.-I. Im, "In vitro chondrogenesis and in vivo repair of osteochondral defect with human induced pluripotent stem cells," Biomaterials, vol. 35, no. 11, pp. 3571-3581, 2014.

[67] D. Cui, H. Li, X. Xu et al., "Mesenchymal stem cells for cartilage regeneration of TMJ osteoarthritis," Stem Cells International, vol. 2017, Article ID 5979741, 11 pages, 2017.

[68] A. K. Dewan, M. A. Gibson, J. H. Elisseeff, and M. E. Trice, "Evolution of autologous chondrocyte repair and comparison to other cartilage repair techniques," BioMed Research International, vol. 2014, Article ID 272481, 11 pages, 2014.

[69] A. Mobasheri, G. Kalamegam, G. Musumeci, and M. E. Batt, "Chondrocyte and mesenchymal stem cell-based therapies for cartilage repair in osteoarthritis and related orthopaedic conditions," Maturitas, vol. 78, no. 3, pp. 188-198, 2014.

[70] J. E. Piletz, J. Drivon, J. Eisenga et al., "Human cells grown with or without substitutes for fetal bovine serum," Cell Medicine, vol. 10, 2018.

[71] S. Yamasaki, K. Nabeshima, Y. Sotomaru et al., "Long-term serial cultivation of mouse induced pluripotent stem cells in serum-free and feeder-free defined medium," The International Journal of Developmental Biology, vol. 57, no. 9-10, pp. 715-724, 2013.

[72] I.-P. Chen, K. Fukuda, N. Fusaki et al., "Induced pluripotent stem cell reprogramming by integration-free Sendai virus vectors from peripheral blood of patients with craniometaphyseal dysplasia," Cellular Reprogramming, vol. 15, no. 6, pp. 503513, 2013. 